\title{
Retention strategies in longitudinal cohort studies: a systematic review and meta- analysis
}

\author{
Samantha Teague1, George J. Youssef ${ }^{1,2}$, Jacqui A. Macdonald ${ }^{1,2,3}$, Emma Sciberras ${ }^{1,2}$, Adrian Shatte 6 , \\ Matthew Fuller-Tyszkiewicz ', Chris Greenwood', Jennifer McIntosh ${ }^{1,2}$, Craig A. Olsson 1,2,3,4, \\ Delyse Hutchinson ${ }^{1,2,3,5^{*}}$ (D) and the SEED Lifecourse Sciences Theme
}

\begin{abstract}
Background: Participant retention strategies that minimise attrition in longitudinal cohort studies have evolved considerably in recent years. This study aimed to assess, via systematic review and meta-analysis, the effectiveness of both traditional strategies and contemporary innovations for retention adopted by longitudinal cohort studies in the past decade.

Methods: Health research databases were searched for retention strategies used within longitudinal cohort studies published in the 10-years prior, with 143 eligible longitudinal cohort studies identified (141 articles; sample size range: 30 to 61,895). Details on retention strategies and rates, research designs, and participant demographics were extracted. Meta-analyses of retained proportions were performed to examine the association between cohort retention rate and individual and thematically grouped retention strategies.

Results: Results identified 95 retention strategies, broadly classed as either: barrier-reduction, community-building, follow-up/reminder, or tracing strategies. Forty-four of these strategies had not been identified in previous reviews. Meta-regressions indicated that studies using barrier-reduction strategies retained $10 \%$ more of their sample $(95 \% \mathrm{Cl}$ [0.13 to 1.08]; $p=.01$ ); however, studies using follow-up/reminder strategies lost an additional $10 \%$ of their sample $(95 \% \mathrm{Cl}[-1.19$ to -0.21$] ; p=.02)$. The overall number of strategies employed was not associated with retention.
\end{abstract}

Conclusions: Employing a larger number of retention strategies may not be associated with improved retention in longitudinal cohort studies, contrary to earlier narrative reviews. Results suggest that strategies that aim to reduce participant burden (e.g., flexibility in data collection methods) might be most effective in maximising cohort retention.

Keywords: Retention, Attrition, Cohort, Longitudinal, Engagement, Follow-up, Drop-out

\section{Background}

Longitudinal cohort studies play a central role in advancing understanding of the onset and progression of physical and mental health problems. Cohort studies assess, and often compare, the incidence of a condition within a group of people who share common characteristics (e.g., being born in the same year) [1]. A key advantage of

\footnotetext{
* Correspondence: delyse.hutchinson@deakin.edu.au

${ }^{1}$ Centre for Social and Early Emotional Development, School of Psychology,

Faculty of Health, Deakin University, Burwood, Geelong, Victoria 3125,

Australia

${ }^{2}$ Murdoch Children's Research Institute, Centre for Adolescent Health, Royal

Children's Hospital, Melbourne, Australia

Full list of author information is available at the end of the article
}

longitudinal cohort studies over other research designs is that repeated measures data temporally orders exposures and outcomes to facilitate causal inference [2]. However, significant and systematic attrition can reduce the generalisability of outcomes and the statistical power to detect effects of interest [3]. Systematic attrition in longitudinal research occurs most often in older, non-white male participants with limited education and/or multiple health problems [4]. Long duration and repeated assessments can also increase attrition due to the significant burden on participants [4]. Given the expense of longitudinal cohort studies, effective strategies that engage and retain cohort

(c) The Author(s). 2018 Open Access This article is distributed under the terms of the Creative Commons Attribution 4.0 International License (http://creativecommons.org/licenses/by/4.0/), which permits unrestricted use, distribution, and reproduction in any medium, provided you give appropriate credit to the original author(s) and the source, provide a link to the Creative Commons license, and indicate if changes were made. The Creative Commons Public Domain Dedication waiver (http://creativecommons.org/publicdomain/zero/1.0/) applies to the data made available in this article, unless otherwise stated. 
participants are critical to the integrity of research outcomes $[5,6]$.

In the last decade, longitudinal data collection methods and cohort retention strategies have evolved considerably. So too have participant expectations of organisations (research and otherwise) that seek information from individuals [7, 8]. Established retention strategies within longitudinal cohort studies include: cash or gift incentives, sending reminder letters to participants, re-sending surveys, and offering alternative methods of data collection (for a review, see [6]). Booker et al. [6] demonstrated that these strategies were effective in longitudinal cohort studies that used the traditional data collection methods of postal surveys, face-to-face visits (home or on-site), and telephone interviews or surveys. However, these cohort retention strategies may not be as well suited to contemporary methods of collecting longitudinal data, such as web and mobile surveys [9], wearable sensors (e.g., FitBits) [10], short message services (SMS) [11], and groupware systems (e.g., video conferencing) [12]. Novel methods of engaging participants such as web advertising [13], social media [14], and electronic reminders [15], are also now being employed in cohort studies using both traditional and modern longitudinal data collection methods.

A systematic review on the effectiveness of established and emerging cohort retention strategies in longitudinal cohort studies would provide guidance to researchers and funders on maximising cohort maintenance within these high investment programs of research. Previous reviews of retention strategies in health research include $[4,6,16,17]$; only one of these reviews focused specifically on longitudinal cohort research designs [6]. Booker et al. [6] conducted a narrative review of retention strategies in longitudinal cohort studies, including incentives, reminders, repeat visits/questionnaires, and alternative methods of data collection, finding that incentives and reminder strategies improved cohort retention. However, this review was limited by the small number of studies identified for each retention strategy, which resulted in the identification of a restricted breadth of retention strategies and the inability to synthesise findings empirically.

Further, Booker et al. [6] did not include research completed after 2006 and thus were unable to investigate emerging cohort retention strategies. Brueton et al. [16] completed a more recent review of retention strategies that included both established and emerging digital data collection retention strategies. However, the authors specifically excluded longitudinal cohort studies and instead focused on participant retention in intervention trials. Differences between intervention and longitudinal cohort studies, such as research design factors (e.g., study duration) and the motivations of the participants in joining or withdrawing from studies, may impact the usefulness of retention strategies across both study designs $[4,6]$.

A review of retention strategies reported in modern longitudinal cohort studies is pertinent and timely, given the emergence of digital retention strategies alongside established retention methods. Maximising cohort retention in longitudinal research can reduce the administration costs of conducting research, improve the efficiency of research processes, and reduce outcome biases for studies by adopting an evidence-based cohort retention framework. In this review, we aimed to: (i) identify retention strategies used in recent longitudinal cohort studies; (ii) examine whether retention rate was moderated by different study or participant characteristics (i.e., number of waves, study duration, sample size, population type, gender, age, country); (iii) estimate the retention rate in studies that use specific retention strategies, and contrast this retention rate with studies that do not use specific retention strategies; (iv) examine whether retention rate is associated with the number of retention strategies used; (v) examine which retention strategies were the strongest independent predictors of retention rate; and (vi) contrast the retention rate based on whether studies utilised emerging or established strategies. Moreover, to ensure that recent innovations in retention strategies were identified, this review focused on literature published within the past 10 years.

\section{Method}

\section{Search strategy}

A systematic review was performed as per the Preferred Reporting Items for Systematic Reviews and Meta-Analyses (PRISMA) guidelines [18]. Two search strategies were implemented. First, the electronic databases Medline, PsycINFO, Embase, CINAHL, AMED, and the Cochrane Library, were searched in July, 2016 using search terms relevant to three themes: (i) attrition, (ii) retention, and (iii) study design (Additional file 1: Table S1). The electronic search was limited to articles published from 2006 onwards in English, to avoid duplication of literature with the previous review on this topic [6]. The search was adapted to suit each database. Second, the reference lists of all articles selected for review were manually searched.

\section{Inclusion and exclusion criteria}

The inclusion and exclusion criteria were determined prior to implementing the search strategy. Articles were included in the review if: (i) the article described a cohort study, which was defined as a representative sample of a group or population who share a common experience or condition [2], (ii) the article reported at least one wave of follow-up data collection with a participant/ proxy, (iii) participant retention data were reported, and (iv) retention strategies were reported. Articles were excluded 
if: (i) the article was not available in English; (ii) the article was not published in a peer-reviewed publication (e.g., conference abstracts or dissertations); and, (iii) the article's research design was cross-sectional, involved data linkage only, or the article was a clinical or non-clinical trial evaluating the effectiveness of treatment regimens or intervention/prevention programmes (for an existing review of retention in intervention studies, see (12)).

\section{Study selection, data extraction, and quality assessment}

The search strategy resulted in 9225 articles after removing duplicates. In total, 141 articles were identified, screened, and determined to be eligible for inclusion (see Fig. 1). Data were extracted and summarised for each of the 141 articles on: (i) the research design, including baseline sample population and sample size, the number of data collection waves reported, and the duration in years between the first and last waves of data collection; (ii) the cohort demographics, including mean sample age at baseline (or age range if mean age was not reported), proportion of male participants, country of cohort participants, and whether the cohort was clinical or non-clinical; and (iii) retention data, including the retention rate between baseline and the final data collection wave reported, and the specific retention strategies. Finally, we examined the suitability of each article in addressing the current study's research question. Articles that listed cohort attrition or retention as a research question or objective were categorised as "retention-focused", and conversely articles that did not focus on attrition or retention were categorised as "non-retention-focused". No articles were excluded on the basis of this quality assessment.

\section{Statistical method}

We used meta-analysis (and meta-regression) to address the aims of the study. Meta-analyses were conducted using the Metafor package v1.9.8 [19] in R software v3.3.1 [20]. The retention rate, defined as the number of individuals who remained in the study at the last wave of data collection as a proportion of the total number of participants recruited at the baseline assessment, was the primary effect size measure of interest. All meta-analyses were conducted using inverse variance weighting, with random effects specified to account for between study heterogeneity. A binomial-normal model (with logit link) was used as the basis for analysis, which is appropriate when the effect size of interest is measured as a proportion. Where appropriate, meta-analytic effects were back-transformed to represent the median meta-analytic retention rate. We also report the $\mathrm{I}^{2}$ statistic as a measure of study heterogeneity, interpreted using the guidelines of Higgins et al. [21]. Meta-analyses were conducted when at least two independent studies contributed to the meta-analysis.
To examine the effect of gender on retention rate, we created a binary variable to denote studies as comprising a higher proportion of either male or female samples (Proportion of male participants in "male" grouping: $M(S D)=73.6 \%(0.20)$; Proportion of female participants in "female" grouping: $M(S D)=75.0 \%(0.21)$ ). To examine the effect of country development level on retention rate, each study country was categorised as either high or low development level by using a mean-split of each nation's Human Development Index - a measure of relative opportunity for longevity, education, and income, with a score range of 0 (low) to 1 (high) (Low HDI group $M(S D)=0.66(0.10)$; High HDI group $M(S D)=$ $0.92(0.02))$ [22]. Retention strategies were coded as either established or emerging, depending on their presence or absence in any of the earlier systematic reviews on participant retention strategies $[4,6,16,17]$. Finally, all meta-regressions adjusted for study duration and number of waves (except when these were specifically examined as predictor variables), given these were deemed to be likely confounding variables in analyses.

\section{Results}

\section{Cohort, participant, and article characteristics}

The 141 articles identified for review described 143 cohorts (41 clinical and 102 non-clinical). Cohorts are summarised in Table 1. Overall the mean sample size reported in the first wave of each article was 3585 participants (range $=30$ to 61,895 ). Articles reported a mean retention rate of $73.5 \%$ $(\mathrm{SD}=20.1 \%)$, with 4.6 waves $(\mathrm{SD}=8.0)$, over 4.3 years $(\mathrm{SD}=$ 5.0). The average baseline participant age was 30.0 years (SD $=22.0$ ), and the average baseline proportion of male participants across samples was $40 \%$ ( $S D=0.30$ ). Studies were conducted in 28 different countries with a mean Human Development Index of $0.79(\mathrm{SD}=0.15)$, indicating that studies were more likely to be conducted in countries with high-levels human development. Cohort attrition/retention was identified as a specific research question or objective of interest in 55 of the 141 articles, indicating that most articles were not focused on participant retention. Retention-focused articles reported significantly more retention strategies than non-retention-focused articles (non-retention-focused: $M(S D)=3.3(3.1)$; retention-focused: $M(S D)=$ $\left.11.0(7.02) ; t_{(141)}=-9.00, p<.001\right)$; however, no differences were found for the study sample size, number of waves, study duration, or retention rate. High heterogeneity was identified in all results, as expected given the diversity of research questions, methodologies, and cohorts across articles [21].

\section{Relationship between retention rate and study or participant characteristics}

To examine whether retention rate was moderated by study characteristics (i.e., number of waves, study duration, 


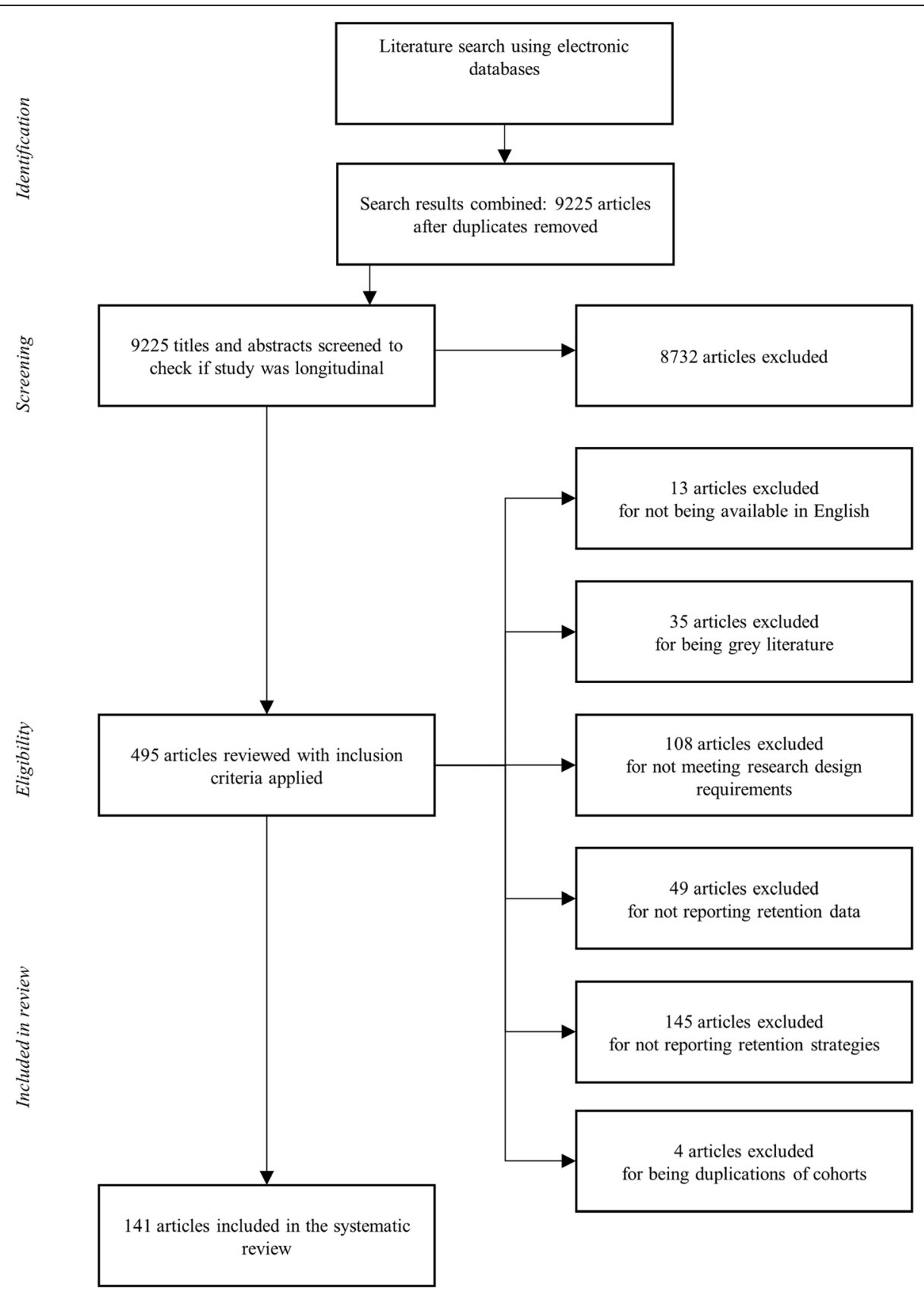

Fig. 1 PRISMA procedural flow chart of the search and identification process

sample size, study focus on retention strategies or not) or by participant characteristics (i.e., population type, gender, age, country development level), a series of meta-regressions was performed, one for each characteristic under examination. Retention rate was not moderated by: number of waves (b $<0.001 ; 95 \%$ CI [ -0.02 to 0.03$], p=.77)$; study duration $(\mathrm{b}=-0.02$; $95 \% \mathrm{CI}[-0.06$ to 0.02$] ; p=.34)$; sample size $(b<-0.001 ; 95 \%$ CI $[-0.00$ to 0.00$] ; p=0.48)$; or articles' focus on retention strategies $(b=-0.12$; $95 \% \mathrm{CI}$ [ -0.54 to 0.30$] ; p=.57$ ). Additionally, retention rate was not associated with the sample characteristics of: cohort type (clinical or non-clinical) $(\mathrm{b}=0.04 ; 95 \% \mathrm{CI}[-0.42$ to -0.51$]$; $p=.86)$; mean age $(\mathrm{b}=0.02 ; 95 \% \mathrm{CI}[-0.01$ to 0.01$] ; p=.74)$; or country development level $(b=0.11 ; 95 \% \mathrm{CI}[-0.46$ to 0.68$] ; p=.71)$. However, gender was a significant moderator of retention rate $(b=-0.67$; $95 \%$ CI $[-1.14$ to -0.20$] ; p<.01))$. Namely, cohorts with more female participants (median retention $=81.5 \%$, 95\% CI [77.6\% to $84.9 \%]$ ) reported higher retention rates than articles with more male participants (median 
Table 1 Description of cohorts reported in the included articles

\begin{tabular}{|c|c|c|c|c|c|c|c|c|}
\hline Sample & Reference & Study Name & $\begin{array}{l}\text { Wave } 1 \\
\text { Sample Size }\end{array}$ & $\begin{array}{l}\text { Wave } 1 \\
\text { Mean Age }\end{array}$ & $\begin{array}{l}\text { Overall } \\
\text { Retention } \\
\text { Rate }\end{array}$ & $\begin{array}{l}\text { No. } \\
\text { Waves }\end{array}$ & $\begin{array}{l}\text { Study } \\
\text { Duration } \\
\text { (years) }\end{array}$ & $\begin{array}{l}\text { No. } \\
\text { Retention } \\
\text { Strategies } \\
\end{array}$ \\
\hline \multicolumn{9}{|l|}{ Clinical cohort studies } \\
\hline $\begin{array}{l}\text { Adolescent and adult } \\
\text { non-injecting heroin } \\
\text { users }\end{array}$ & {$[32]$} & Project Brown & 300 & $16-40$ & $98 \%$ & 2 & 1.00 & 9 \\
\hline $\begin{array}{l}\text { Adolescent/young } \\
\text { adult cancer patients }\end{array}$ & [33] & $\begin{array}{l}\text { Resilience in Adolescents and } \\
\text { Young Adults with Cancer Study }\end{array}$ & 52 & 17.6 & $35 \%$ & 3 & 1.50 & 4 \\
\hline $\begin{array}{l}\text { Adolescent/Young } \\
\text { Adult mobile young } \\
\text { injection drug users }\end{array}$ & {$[34]$} & & 101 & 22 & $48 \%$ & 6 & 2.00 & 9 \\
\hline $\begin{array}{l}\text { Adolescents and } \\
\text { young adults with } \\
\text { Type } 1 \text { Diabetes }\end{array}$ & {$[35]$} & $\begin{array}{l}\text { Young Adult Diabetes Assessment } \\
\text { (YADA) }\end{array}$ & 204 & $17-18$ & $97 \%$ & 3 & 5.00 & 18 \\
\hline $\begin{array}{l}\text { Adult asthmatic } \\
\text { pregnant women }\end{array}$ & {$[36]$} & $\begin{array}{l}\text { Syracuse AUDIT (Assessment of } \\
\text { Urban Dwellings for Indoor Toxics) }\end{array}$ & 103 & 25.4 & $86 \%$ & 5 & 1.00 & 9 \\
\hline Adult cannabis users & {$[37]$} & & 193 & 32 & $84 \%$ & 90 & 0.25 & 3 \\
\hline $\begin{array}{l}\text { Adult entitlement } \\
\text { claimants from } \\
\text { the Accident } \\
\text { Compensation } \\
\text { Corporation }\end{array}$ & {$[38]$} & $\begin{array}{l}\text { Prospective Outcomes of Injury Study } \\
\text { (POIS) }\end{array}$ & 2856 & $18-64$ & $79 \%$ & 4 & 2.00 & 4 \\
\hline $\begin{array}{l}\text { Adult major } \\
\text { trauma patients }\end{array}$ & {$[39]$} & Victorian State Trauma Registry & 1102 & 40 & $70 \%$ & 2 & 0.50 & 3 \\
\hline $\begin{array}{l}\text { Adult myocardial } \\
\text { infarction survivors }\end{array}$ & {$[40]$} & $\begin{array}{l}\text { Western New York Acute Myocardial } \\
\text { Infarction (MI) Study }\end{array}$ & 884 & 54 & $90 \%$ & 4 & 7.00 & 2 \\
\hline $\begin{array}{l}\text { Adult parents of } \\
\text { overweight children } \\
\text { with low-income }\end{array}$ & {$[41]$} & & 37 & $20-50+$ & $46 \%$ & 26 & 1.00 & 14 \\
\hline $\begin{array}{l}\text { Adult Puerto Rican/ } \\
\text { Mexicans with a } \\
\text { mental health } \\
\text { diagnosis }\end{array}$ & {$[42]$} & & 68 & $18-50$ & $59 \%$ & 3 & 5.00 & 20 \\
\hline $\begin{array}{l}\text { Adult smokers } \\
\text { and non-smoker } \\
\text { comparisons }\end{array}$ & {$[43]$} & $\begin{array}{l}\text { International Tobacco Control (ITC) China } \\
\text { Survey }\end{array}$ & 6001 & $18-55+$ & $68 \%$ & 3 & 3.00 & 7 \\
\hline $\begin{array}{l}\text { Adult spinal } \\
\text { surgery patients }\end{array}$ & {$[44]$} & Danish spine surgery registry (Danespine) & 506 & 58.94 & $100 \%$ & 3 & 1.00 & 1 \\
\hline $\begin{array}{l}\text { Adult survivors } \\
\text { of ARDS }\end{array}$ & {$[45]$} & $\begin{array}{l}\text { Toronto Acute Respiratory Distress } \\
\text { Syndrome (ARDS) Study }\end{array}$ & 109 & - & $86 \%$ & 3 & 5.00 & 18 \\
\hline $\begin{array}{l}\text { Adult survivors } \\
\text { of SARS }\end{array}$ & {$[45]$} & $\begin{array}{l}\text { Toronto Severe acute respiratory } \\
\text { syndrome (SARS) Study }\end{array}$ & 117 & - & $91 \%$ & 2 & 2.00 & 17 \\
\hline Adults with diabetes & {$[46]$} & Living with Diabetes Study & 3951 & 61.4 & $81 \%$ & 3 & 3.00 & 15 \\
\hline $\begin{array}{l}\text { Adult women at-risk } \\
\text { of cardiovascular } \\
\text { events }\end{array}$ & {$[47]$} & PREDICT Study & 1110 & $21+$ & $90 \%$ & 9 & 2.00 & 12 \\
\hline $\begin{array}{l}\text { Adult women at-risk } \\
\text { of HIV infection }\end{array}$ & {$[48]$} & & 411 & 21 & $94 \%$ & 2 & 1.00 & 4 \\
\hline $\begin{array}{l}\text { Adult women breast } \\
\text { cancer survivors }\end{array}$ & [49] & & 121 & 59.7 & $96 \%$ & 2 & 1.00 & 3 \\
\hline $\begin{array}{l}\text { Adult women } \\
\text { with HIV/AIDS }\end{array}$ & {$[50]$} & $\begin{array}{l}\text { Instituto de Pesquisa Clínica Evandro } \\
\text { Chagas (IPEC) Cohort of Women Living } \\
\text { with HIV/ AIDS followed up in Fundação } \\
\text { Oswaldo Cruz (FIOCRUZ) Rio de Janeiro }\end{array}$ & 225 & 32 & $56 \%$ & 3 & 3.00 & 2 \\
\hline $\begin{array}{l}\text { Adults at first-episode } \\
\text { psychosis }\end{array}$ & {$[51]$} & & 71 & $18-60$ & $70 \%$ & 3 & 5.00 & 1 \\
\hline
\end{tabular}


Table 1 Description of cohorts reported in the included articles (Continued)

\begin{tabular}{|c|c|c|c|c|c|c|c|c|}
\hline Sample & Reference & Study Name & $\begin{array}{l}\text { Wave } 1 \\
\text { Sample Size }\end{array}$ & $\begin{array}{l}\text { Wave } 1 \\
\text { Mean Age }\end{array}$ & $\begin{array}{l}\text { Overall } \\
\text { Retention } \\
\text { Rate } \\
\end{array}$ & $\begin{array}{l}\text { No. } \\
\text { Waves }\end{array}$ & $\begin{array}{l}\text { Study } \\
\text { Duration } \\
\text { (years) }\end{array}$ & $\begin{array}{l}\text { No. } \\
\text { Retention } \\
\text { Strategies } \\
\end{array}$ \\
\hline $\begin{array}{l}\text { Adults at-risk for } \\
\text { HIV infection }\end{array}$ & {$[52]$} & & 2191 & $18-49$ & $77 \%$ & 2 & 1.00 & 4 \\
\hline $\begin{array}{l}\text { Adults at-risk of } \\
\text { problem gambling } \\
\text { plus comparison } \\
\text { group }\end{array}$ & [53] & Quinte Longitudinal Study & 4121 & 46.1 & $94 \%$ & 5 & 5.00 & 1 \\
\hline Adults who self-harm & [54] & & 150 & 28.4 & $95 \%$ & 3 & 6.00 & 4 \\
\hline $\begin{array}{l}\text { Adults who use } \\
\text { urinary catheters }\end{array}$ & [55] & & 33 & 43 & $100 \%$ & 4 & 0.50 & 2 \\
\hline $\begin{array}{l}\text { Adults with acute } \\
\text { transient ischemic } \\
\text { attack or stroke }\end{array}$ & [56] & Oxford Vascular Study & 1236 & 75.2 & $98 \%$ & 7 & 10.00 & 3 \\
\hline $\begin{array}{l}\text { Adults with } \\
\text { Alzheimers Disease }\end{array}$ & {$[57]$} & REAL.FR study & 686 & 77.9 & $59 \%$ & 2 & 2.00 & 3 \\
\hline $\begin{array}{l}\text { Adults with Alzheimers } \\
\text { Disease }(A D) \text { and } \\
\text { their carers }\end{array}$ & {$[58]$} & & 40 & 78 & $81 \%$ & 5 & 1.00 & 16 \\
\hline $\begin{array}{l}\text { Adults with Alzheimers } \\
\text { Disease or Mild } \\
\text { Cognitive Impairment } \\
\text { and comparison }\end{array}$ & [59] & $\begin{array}{l}\text { Australian Imaging, Biomarkers and } \\
\text { Lifestyle Flagship Study of Ageing (AIBL) }\end{array}$ & 1112 & 69.7 & $90 \%$ & 2 & 1.50 & 3 \\
\hline $\begin{array}{l}\text { Adults with } \\
\text { aneurysmal } \\
\text { subarachnoid } \\
\text { hemorrhage (aSAH) }\end{array}$ & {$[60]$} & Family Caregiver study & 59 & 52 & $83 \%$ & 4 & 1.00 & 5 \\
\hline Adults with back pain & {$[61]$} & & 250 & $30-59$ & $68 \%$ & 14 & 7.00 & 3 \\
\hline $\begin{array}{l}\text { Adults with primary } \\
\text { malignant brain } \\
\text { tumour (PMBT) and } \\
\text { their caregivers }\end{array}$ & {$[60]$} & 20-Hete Study & 496 & 53.12 & $90 \%$ & 3 & 1.00 & 3 \\
\hline $\begin{array}{l}\text { Adults with primary } \\
\text { Sjögren's syndrome }\end{array}$ & {$[62]$} & & 222 & 52.5 & $70 \%$ & 2 & 7.60 & 2 \\
\hline $\begin{array}{l}\text { Adults with } \\
\text { schizophrenia and } \\
\text { comparison group }\end{array}$ & [63] & & 56 & 21 & $89 \%$ & 2 & 2.00 & 1 \\
\hline $\begin{array}{l}\text { Adults with Severe } \\
\text { Traumatic Brain Injury }\end{array}$ & [64] & PariS-TBI study & 504 & 42 & $60 \%$ & 2 & 4.00 & 6 \\
\hline $\begin{array}{l}\text { Adults with } \\
\text { temporomandibular } \\
\text { disorders }\end{array}$ & [65] & $\begin{array}{l}\text { Orofacial Pain: Prospective Evaluation } \\
\text { and Risk Assessment (OPPERA) Study }\end{array}$ & 3263 & 31 & $84 \%$ & 11 & 2.80 & 5 \\
\hline $\begin{array}{l}\text { Adults with traumatic } \\
\text { brain injury }\end{array}$ & [66] & Tasmanian Neurotrauma Register (TNTR) & 947 & 36.1 & $19 \%$ & 7 & 3.00 & 5 \\
\hline $\begin{array}{l}\text { Adults with Traumatic } \\
\text { Brain Injury }\end{array}$ & {$[67]$} & & 77 & 67.1 & $57 \%$ & 3 & 0.50 & 1 \\
\hline Adult burn victims & {$[68]$} & $\begin{array}{l}\text { Burns Registry of Australia and } \\
\text { New Zealand }\end{array}$ & 463 & 41.8 & $21 \%$ & 5 & 2.00 & 4 \\
\hline $\begin{array}{l}\text { Caregivers of adult } \\
\text { cancer patients }\end{array}$ & [69] & & 206 & 57 & $85 \%$ & 3 & 1.10 & 1 \\
\hline $\begin{array}{l}\text { Child twins and } \\
\text { their siblings }\end{array}$ & {$[70]$} & Australian Twin ADHD Project (ATAP) & 1938 & $4-12$ & $43 \%$ & 3 & 9.00 & 3 \\
\hline $\begin{array}{l}\text { Children at-risk of } \\
\text { HIV infection }\end{array}$ & {$[71]$} & AIDS-ill families study & 3515 & 13.5 & $97 \%$ & 2 & 1.00 & 9 \\
\hline $\begin{array}{l}\text { Children at-risk of } \\
\text { thyroid cancer and }\end{array}$ & {$[72]$} & & 600 & 11 & $88 \%$ & 2 & 8.00 & 9 \\
\hline
\end{tabular}


Table 1 Description of cohorts reported in the included articles (Continued)

\begin{tabular}{|c|c|c|c|c|c|c|c|c|}
\hline Sample & Reference & Study Name & $\begin{array}{l}\text { Wave } 1 \\
\text { Sample Size }\end{array}$ & $\begin{array}{l}\text { Wave } 1 \\
\text { Mean Age }\end{array}$ & $\begin{array}{l}\text { Overall } \\
\text { Retention } \\
\text { Rate } \\
\end{array}$ & $\begin{array}{l}\text { No. } \\
\text { Waves }\end{array}$ & $\begin{array}{l}\text { Study } \\
\text { Duration } \\
\text { (years) }\end{array}$ & $\begin{array}{l}\text { No. } \\
\text { Retention } \\
\text { Strategies } \\
\end{array}$ \\
\hline $\begin{array}{l}\text { Children exposed to } \\
\text { Cocaine/opiate } \\
\text { and comparison }\end{array}$ & {$[73]$} & Maternal Lifestyle Study (MLS) & 13,888 & 0.1 & $76 \%$ & 5 & 15.00 & 16 \\
\hline $\begin{array}{l}\text { Children perinatally } \\
\text { infected with HIV } \\
\text { and comparison }\end{array}$ & [74] & $\begin{array}{l}\text { IMPAACT P1055 Psychiatric } \\
\text { Co-Morbidity Study }\end{array}$ & 582 & 12.4 & $81 \%$ & 2 & 2.00 & 3 \\
\hline $\begin{array}{l}\text { Children who were } \\
\text { former child soldiers }\end{array}$ & [75] & & 260 & $10-17$ & $69 \%$ & 3 & 6.00 & 3 \\
\hline $\begin{array}{l}\text { Children with ADHD } \\
\text { and a sibling } \\
\text { for comparison }\end{array}$ & [26] & $\begin{array}{l}\text { International Multicenter ADHD } \\
\text { Genetics (IMAGE) study }\end{array}$ & 459 & 11.4 & $76 \%$ & 2 & 6.00 & 1 \\
\hline $\begin{array}{l}\text { Children with ADHD } \\
\text { and comparisons }\end{array}$ & {$[76]$} & $\begin{array}{l}\text { Berkeley Girls with ADHD } \\
\text { Longitudinal Study (BGALS) }\end{array}$ & 228 & 9.6 & $95 \%$ & 3 & 10.00 & 2 \\
\hline $\begin{array}{l}\text { Female adolescent/ } \\
\text { young adult survivors } \\
\text { of a mass campus } \\
\text { shooting }\end{array}$ & {$[77]$} & & 812 & 19 & $81 \%$ & 7 & 2.50 & 1 \\
\hline $\begin{array}{l}\text { Infants at-risk of } \\
\text { developing diabetes }\end{array}$ & {$[78]$} & $\begin{array}{l}\text { The Environmental Determinants of } \\
\text { Diabetets in the Young (TEDDY) study }\end{array}$ & 4138 & 0.4 & $74 \%$ & 3 & 1.00 & 1 \\
\hline Male sex workers & {$[79]$} & & 50 & $17-26+$ & $34 \%$ & 2 & 0.50 & 4 \\
\hline $\begin{array}{l}\text { Men who have } \\
\text { Sex with Men }\end{array}$ & {$[25]$} & $\begin{array}{l}\text { Bangkok Men who have Sex with } \\
\text { Men Cohort Study (BMCS) }\end{array}$ & 1744 & 26 & $90 \%$ & 10 & 3.00 & 3 \\
\hline $\begin{array}{l}\text { Men who have } \\
\text { Sex with Men }\end{array}$ & {$[80]$} & & 2607 & 22.7 & $22 \%$ & 2 & 0.25 & 2 \\
\hline $\begin{array}{l}\text { Men who have } \\
\text { Sex with Men }\end{array}$ & {$[81]$} & & 710 & $18-54$ & $74 \%$ & 2 & 1.00 & 5 \\
\hline $\begin{array}{l}\text { Men who have } \\
\text { Sex with Men }\end{array}$ & {$[82]$} & & 1003 & 28 & $70 \%$ & 8 & 2.60 & 1 \\
\hline $\begin{array}{l}\text { Men who have } \\
\text { Sex with Men }\end{array}$ & {$[83]$} & & 511 & 29 & $55 \%$ & 3 & 0.75 & 5 \\
\hline $\begin{array}{l}\text { Men who have } \\
\text { Sex with Men }\end{array}$ & {$[84]$} & & 278 & 32 & $16 \%$ & 3 & 1.00 & 5 \\
\hline $\begin{array}{l}\text { Men who have } \\
\text { Sex with Men }\end{array}$ & {$[85]$} & & 327 & 30.8 & $92 \%$ & 3 & 1.00 & 1 \\
\hline $\begin{array}{l}\text { Population at-risk } \\
\text { for HIV infection }\end{array}$ & {$[86]$} & & 1000 & $13-49$ & $77 \%$ & 5 & 2.50 & 3 \\
\hline \multicolumn{9}{|l|}{ Non-clinical cohort studies } \\
\hline $\begin{array}{l}\text { Adolescent } \\
\text { mother-child dyads }\end{array}$ & {$[87]$} & & 97 & $14-20$ & $38 \%$ & 3 & 4.00 & 9 \\
\hline Adolescent population & {$[88]$} & Danish Youth Cohort & 12,498 & 13.4 & $25 \%$ & 3 & 2.00 & 1 \\
\hline Adolescent population & {$[89]$} & Dating It Safe & 964 & 16.1 & $86 \%$ & 2 & 1.00 & 1 \\
\hline Adolescent population & {$[90]$} & Healthy Teens Longitudinal Study & 611 & 14.8 & $66 \%$ & 7 & 6.00 & 1 \\
\hline Adolescent population & {$[91]$} & $\begin{array}{l}\text { International Youth Development } \\
\text { Study (IYDS) }\end{array}$ & 1858 & 13 & $98 \%$ & 3 & 2.00 & 4 \\
\hline Adolescent population & {$[92]$} & $\begin{array}{l}\text { TRacking Adolescents' Individual } \\
\text { Lives Survey (TRAILS) }\end{array}$ & 2773 & 11.1 & $79 \%$ & 4 & 8.00 & 1 \\
\hline Adolescent population & [93] & Youth Asset Study (YAS) & 1117 & $12-17$ & $97 \%$ & 5 & 4.00 & 32 \\
\hline Adolescent population & [94] & & 1535 & 14.9 & $57 \%$ & 2 & 1.00 & 1 \\
\hline Adolescent population & {$[95]$} & & 497 & 13.03 & $86 \%$ & 6 & 6.00 & 1 \\
\hline $\begin{array}{l}\text { Adolescent/Young } \\
\text { adult twins }\end{array}$ & [96] & Minnesota Twin Family Study (MTFS) & 1252 & 17 & $93 \%$ & 4 & 12.00 & 1 \\
\hline
\end{tabular}


Table 1 Description of cohorts reported in the included articles (Continued)

\begin{tabular}{|c|c|c|c|c|c|c|c|c|}
\hline Sample & Reference & Study Name & $\begin{array}{l}\text { Wave } 1 \\
\text { Sample Size }\end{array}$ & $\begin{array}{l}\text { Wave } 1 \\
\text { Mean Age }\end{array}$ & $\begin{array}{l}\text { Overall } \\
\text { Retention } \\
\text { Rate }\end{array}$ & $\begin{array}{l}\text { No. } \\
\text { Waves }\end{array}$ & $\begin{array}{l}\text { Study } \\
\text { Duration } \\
\text { (years) }\end{array}$ & $\begin{array}{l}\text { No. } \\
\text { Retention } \\
\text { Strategies } \\
\end{array}$ \\
\hline $\begin{array}{l}\text { Adult African } \\
\text { American population }\end{array}$ & [97] & $\begin{array}{l}\text { Religion and Health in African Americans } \\
\text { (RHIAA) study }\end{array}$ & 2803 & 54.86 & $40 \%$ & 2 & 2.50 & 16 \\
\hline $\begin{array}{l}\text { Adult African } \\
\text { American women }\end{array}$ & [98] & $\begin{array}{l}\text { Study of Environment, Lifestyle and } \\
\text { Fibroids (SELF) }\end{array}$ & 1696 & $23-34$ & $87 \%$ & 2 & 1.67 & 3 \\
\hline $\begin{array}{l}\text { Adult Alaska Native } \\
\text { and American } \\
\text { Indian population }\end{array}$ & [99] & $\begin{array}{l}\text { Education and Research Towards Health } \\
\text { (EARTH) study }\end{array}$ & 3828 & $18-55+$ & $88 \%$ & 2 & 1.50 & 18 \\
\hline $\begin{array}{l}\text { Adult low } \\
\text { income mothers }\end{array}$ & {$[100]$} & Welfare Client Longitudinal Study (WCLS) & 498 & $18-35+$ & $89 \%$ & 2 & 1.00 & 11 \\
\hline Adult male population & [101] & $\begin{array}{l}\text { Florey Adelaide Male Ageing Study } \\
\text { (FAMAS) }\end{array}$ & 1195 & 55 & $96 \%$ & 2 & 1.00 & 14 \\
\hline $\begin{array}{l}\text { Adult mother-child } \\
\text { dyads }\end{array}$ & [102] & & 4318 & 0.2 & $84 \%$ & 5 & 1.00 & 3 \\
\hline $\begin{array}{l}\text { Adult mother-child } \\
\text { dyads }\end{array}$ & [103] & & 365 & 13.7 & $64 \%$ & 2 & 1.00 & 4 \\
\hline Adult officeworkers & [104] & & 53 & 42 & $100 \%$ & 26 & 1.00 & 1 \\
\hline $\begin{array}{l}\text { Adult online panel } \\
\text { members }\end{array}$ & [105] & ATTEMPT Cohort & 2009 & 47.9 & $52 \%$ & 5 & 1.00 & 3 \\
\hline $\begin{array}{l}\text { Adult online panel } \\
\text { members }\end{array}$ & [106] & & 202 & 33.8 & $47 \%$ & 3 & 0.00 & 1 \\
\hline Adult population & [107] & $\begin{array}{l}\text { Baltimore Epidemiologic Catchment } \\
\text { Area Follow-up }\end{array}$ & 3481 & $18-65+$ & $53 \%$ & 3 & 23.00 & 1 \\
\hline Adult population & [108] & $\begin{array}{l}\text { Healthy Aging in Neighborhoods of } \\
\text { Diversity across the Life Span } \\
\text { (HANDLS) study }\end{array}$ & 3722 & $30-64$ & $79 \%$ & 3 & 4.00 & 12 \\
\hline Adult population & [109] & $\begin{array}{l}\text { Heart Strategies Concentrating on } \\
\text { Risk Evaluation (Heart SCORE) study }\end{array}$ & 1841 & 59.1 & $84 \%$ & 5 & 4.00 & 11 \\
\hline Adult population & [110] & Helsinki Aging Study (HAS) & 170 & 80 & $42 \%$ & 2 & 5.00 & 3 \\
\hline Adult population & [111] & Knee Clinical Assessment Study (CAS(K)) & 819 & $50-80+$ & $95 \%$ & 2 & 1.50 & 3 \\
\hline Adult population & [112] & Longitudinal Assessment of Women (LAW) & 511 & 64.7 & $96 \%$ & 5 & 5.00 & 16 \\
\hline Adult population & [113] & Midlife in the United States (MIDUS) & 7108 & $25-74$ & $75 \%$ & 2 & 10.00 & 4 \\
\hline Adult population & [114] & $\begin{array}{l}\text { MRC National Survey of Health } \\
\text { and Development (NSHD) }\end{array}$ & 3163 & $60-64$ & $84 \%$ & 2 & 9.00 & 2 \\
\hline Adult population & [115] & $\begin{array}{l}\text { Netherlands Mental Health Survey } \\
\text { and Incidence Study (NEMESIS-2) }\end{array}$ & $18-64$ & $18+$ & $80 \%$ & 2 & 3.00 & 9 \\
\hline Adult population & [116] & $\begin{array}{l}\text { Netherlands Study of Depression } \\
\text { and Anxiety (NESDA) }\end{array}$ & 2981 & 39.9 & $87 \%$ & 2 & 2.00 & 10 \\
\hline Adult population & [117] & New Zealand Attitudes and Values Study & 6518 & 48 & $62 \%$ & 4 & 3.00 & 12 \\
\hline Adult population & [118] & NutriNet-Santé Cohort Study & 15,000 & $18+$ & $44 \%$ & 2 & 2.00 & 8 \\
\hline Adult population & [119] & $\begin{array}{l}\text { People's Republic of China-United States } \\
\text { of America (PRC-USA) Collaborative Study } \\
\text { of Cardiovascular and Cardiopulmonary } \\
\text { Epidemiology }\end{array}$ & 1739 & 57.7 & $94 \%$ & 3 & 5.00 & 1 \\
\hline Adult population & [120] & Quinte Longitudinal Study (QLS) & 4121 & $18-65+$ & $94 \%$ & 5 & 5.00 & 1 \\
\hline Adult population & [121] & Study of health in Pomerania (SHIP) & 6267 & $20-79$ & $84 \%$ & 2 & 5.00 & 10 \\
\hline Adult population & [122] & $\begin{array}{l}\text { Study of Use of Products and } \\
\text { Exposure-Related Behavior (SUPERB) }\end{array}$ & 481 & 36 & $47 \%$ & 9 & 3.00 & 3 \\
\hline Adult population & [123] & & 700 & 48.8 & $71 \%$ & 4 & 2.00 & 2 \\
\hline $\begin{array}{l}\text { Adult pregnant } \\
\text { women }\end{array}$ & [124] & Drakenstein Child Health Study (DCHS) & 585 & 26.6 & $90 \%$ & 2 & 1.33 & 6 \\
\hline
\end{tabular}


Table 1 Description of cohorts reported in the included articles (Continued)

\begin{tabular}{|c|c|c|c|c|c|c|c|c|}
\hline Sample & Reference & Study Name & $\begin{array}{l}\text { Wave } 1 \\
\text { Sample Size }\end{array}$ & $\begin{array}{l}\text { Wave } 1 \\
\text { Mean Age }\end{array}$ & $\begin{array}{l}\text { Overall } \\
\text { Retention } \\
\text { Rate }\end{array}$ & $\begin{array}{l}\text { No. } \\
\text { Waves }\end{array}$ & $\begin{array}{l}\text { Study } \\
\text { Duration } \\
\text { (years) }\end{array}$ & $\begin{array}{l}\text { No. } \\
\text { Retention } \\
\text { Strategies } \\
\end{array}$ \\
\hline $\begin{array}{l}\text { Adult pregnant } \\
\text { women }\end{array}$ & [125] & G-GrippeNet (GGNET) Project & 153 & 34 & $78 \%$ & 10 & 0.20 & 2 \\
\hline $\begin{array}{l}\text { Adult pregnant } \\
\text { women }\end{array}$ & {$[126]$} & $\begin{array}{l}\text { Maternal Anxiety in Relation to Infant } \\
\text { Development (MARI) Study }\end{array}$ & 306 & 28 & $90 \%$ & 7 & 2.00 & 2 \\
\hline $\begin{array}{l}\text { Adult pregnant } \\
\text { women }\end{array}$ & {$[127]$} & $\begin{array}{l}\text { Mater-University Study of Pregnancy } \\
\text { (MUSP) }\end{array}$ & 6753 & 24.3 & $88 \%$ & 6 & 27.00 & 5 \\
\hline $\begin{array}{l}\text { Adult pregnant } \\
\text { women }\end{array}$ & {$[128]$} & Pregnancy, Infection, and Nutrition Study & 262 & 30 & $70 \%$ & 2 & 2.00 & 5 \\
\hline $\begin{array}{l}\text { Adult pregnant } \\
\text { women }\end{array}$ & {$[129]$} & & 118 & 31.6 & $72 \%$ & 4 & 1.00 & 1 \\
\hline $\begin{array}{l}\text { Adult pregnant } \\
\text { women }\end{array}$ & [130] & & 40,333 & 30.3 & $65 \%$ & 2 & 0.75 & 1 \\
\hline $\begin{array}{l}\text { Adult pregnant } \\
\text { women }\end{array}$ & [131] & & 1040 & $18-34+$ & $71 \%$ & 3 & 1.10 & 1 \\
\hline $\begin{array}{l}\text { Adult premenopausal } \\
\text { women }\end{array}$ & [132] & Uterine Fibroid Study (UFS) & 1141 & $35-49$ & $85 \%$ & 3 & 8.00 & 5 \\
\hline $\begin{array}{l}\text { Adult South Asians } \\
\text { living in US }\end{array}$ & [133] & $\begin{array}{l}\text { Mediators of Atherosclerosis in South } \\
\text { Asians Living in America (MASALA) study }\end{array}$ & 906 & $40-84$ & $48 \%$ & 2 & 0.75 & 6 \\
\hline Adult veterans & [134] & & 1319 & 33 & $79 \%$ & 2 & 1.00 & 4 \\
\hline Adult women & {$[135]$} & $\begin{array}{l}\text { Australian Longitudinal Study on } \\
\text { Women's Health }\end{array}$ & 14,247 & $18-23$ & $77 \%$ & 4 & 4.00 & 8 \\
\hline Adult women & {$[136]$} & $\begin{array}{l}\text { Australian Longitudinal Study on } \\
\text { Women's Health }\end{array}$ & 40,395 & $18-75$ & $80 \%$ & 2 & 6.00 & 5 \\
\hline Adult women & [137] & Manitoba Breast Screening Program & 47,637 & $50-68$ & $80 \%$ & 2 & 2.50 & 5 \\
\hline Adult women & [138] & & 1435 & $40-50$ & $72 \%$ & 3 & 3.00 & 13 \\
\hline Adult women & [139] & & 48,125 & 38 & $91 \%$ & 2 & 12.00 & 5 \\
\hline $\begin{array}{l}\text { Adult women hoping } \\
\text { to become pregnant }\end{array}$ & [140] & & 30 & 29.4 & $43 \%$ & 4 & 1.50 & 2 \\
\hline $\begin{array}{l}\text { Adult/Young Adult } \\
\text { Probationers }\end{array}$ & [141] & & 199 & $17-35$ & $52 \%$ & 5 & 15.00 & 7 \\
\hline Birth cohort & {$[142]$} & Australian Aboriginal Birth Cohort study & 686 & 0 & $72 \%$ & 3 & 18.00 & 31 \\
\hline Birth cohort & {$[143]$} & Birth to Twenty (BT20) birth cohort & 3273 & 0 & $70 \%$ & 19 & 16.00 & 16 \\
\hline Birth cohort & {$[144]$} & Danish National Birth Cohort & 61,895 & 0 & $63 \%$ & 2 & 7.00 & 1 \\
\hline Birth cohort & [145] & $\begin{array}{l}\text { ECAGE Project (Study of Food Intake and } \\
\text { Eating Behavior of Pregnant Women) }\end{array}$ & 462 & 0 & $94 \%$ & 3 & 0.65 & 2 \\
\hline Birth cohort & {$[146]$} & Environments for Healthy Living (EHL) & 3368 & 0 & $65 \%$ & 2 & 5.50 & 7 \\
\hline Birth cohort & [147] & $\begin{array}{l}\text { Geographic research on wellbeing } \\
\text { (GROW) study }\end{array}$ & 9256 & 7 & $33 \%$ & 2 & 7.00 & 9 \\
\hline Birth cohort & {$[148]$} & Growing up in New Zealand & 6846 & 0 & $95 \%$ & 2 & 0.75 & 23 \\
\hline Birth cohort & [149] & Japan Children's Study (JCS) & 467 & 0.3 & $81 \%$ & 6 & 3.50 & 13 \\
\hline Birth cohort & [150] & $\begin{array}{l}\text { Nascita e INFanzia gli Effetti } \\
\text { dell'Ambiente (NINFEA) cohort }\end{array}$ & 7003 & 0 & $78 \%$ & 4 & 4.00 & 6 \\
\hline Birth cohort & [151] & & 413 & 0 & $95 \%$ & 2 & 0.50 & 1 \\
\hline Birth cohort & [152] & & 1196 & 0 & $46 \%$ & 5 & 30.00 & 1 \\
\hline $\begin{array}{l}\text { Birth cohort of } \\
\text { children from } \\
\text { Lesbian parents }\end{array}$ & [153] & $\begin{array}{l}\text { US National Longitudinal Lesbian } \\
\text { Family Study (NLLFS) }\end{array}$ & 154 & 0 & $93 \%$ & 5 & 17.00 & 1 \\
\hline $\begin{array}{l}\text { Child African-American } \\
\text { population and }\end{array}$ & [154] & & 76 & 3.4 & $70 \%$ & 2 & 3.50 & 18 \\
\hline
\end{tabular}


Table 1 Description of cohorts reported in the included articles (Continued)

\begin{tabular}{|c|c|c|c|c|c|c|c|c|}
\hline Sample & Reference & Study Name & $\begin{array}{l}\text { Wave } 1 \\
\text { Sample Size }\end{array}$ & $\begin{array}{l}\text { Wave } 1 \\
\text { Mean Age }\end{array}$ & $\begin{array}{l}\text { Overall } \\
\text { Retention } \\
\text { Rate }\end{array}$ & $\begin{array}{l}\text { No. } \\
\text { Waves }\end{array}$ & $\begin{array}{l}\text { Study } \\
\text { Duration } \\
\text { (years) }\end{array}$ & $\begin{array}{l}\text { No. } \\
\text { Retention } \\
\text { Strategies }\end{array}$ \\
\hline $\begin{array}{l}\text { Child monozygotic (MZ) } \\
\text { and dizygotic (DZ) twins }\end{array}$ & [155] & $\begin{array}{l}\text { University of Southern California Study of } \\
\text { Risk Factors for Antisocial Behavior (USC RFAB) }\end{array}$ & 1569 & 9 & $59 \%$ & 5 & 8.00 & 10 \\
\hline Child population & {$[156]$} & Danish youth cohort Vestliv & 3054 & 14.5 & $64 \%$ & 3 & 6.00 & 1 \\
\hline Child population & [157] & Ho Chi Minh City Youth Cohort & 759 & 11.8 & $77 \%$ & 5 & 5.00 & 4 \\
\hline Child population & [158] & & 405 & 11 & $91 \%$ & 4 & 4.00 & 17 \\
\hline $\begin{array}{l}\text { Indigenous } \\
\text { adolescents }\end{array}$ & [159] & & 671 & 11.3 & $79 \%$ & 8 & 8.00 & 7 \\
\hline Mother-child dyads & [160] & $\begin{array}{l}\text { Center for Oral Health Research in } \\
\text { Appalachia } 2 \text { (COHRA2) Study }\end{array}$ & 744 & 28.4 & $79 \%$ & 2 & 2.50 & 1 \\
\hline Older adults & [161] & Cardiovascular Health Study (CHS) & 5888 & 73 & $46 \%$ & 2 & 7.00 & 2 \\
\hline Older adults & [162] & $\begin{array}{l}\text { Chinese Longitudinal Healthy Longevity } \\
\text { Survey (CLHLS) }\end{array}$ & 16,020 & $65+$ & $56 \%$ & 3 & 2.00 & 1 \\
\hline Older adults & [163] & $\begin{array}{l}\text { Longitudinal Aging Study Amsterdam } \\
\text { (LASA) }\end{array}$ & 3107 & 70 & $32 \%$ & 6 & 17.00 & 6 \\
\hline Older adults & [164] & New England Centenarian Study (NECS) & 759 & $97+$ & $86 \%$ & 2 & 3.50 & 1 \\
\hline Older adults & [165] & Newcastle $85+$ Study & 854 & $85+$ & $40 \%$ & 4 & 5.00 & 11 \\
\hline Older adults & [166] & $\begin{array}{l}\text { Physiological Research to Improve Sleep } \\
\text { and Memory Project }\end{array}$ & 78.2 & $70+$ & $83 \%$ & 3 & 2.00 & 24 \\
\hline Older adults & [167] & UAB Study of Aging & 1000 & $65+$ & $95 \%$ & 2 & 4.00 & 16 \\
\hline $\begin{array}{l}\text { Population during } \\
\text { political turmoil }\end{array}$ & [168] & & 889 & 36 & $89 \%$ & 2 & 0.50 & 1 \\
\hline $\begin{array}{l}\text { Population during } \\
\text { political turmoil }\end{array}$ & [169] & & 1022 & 33.9 & $85 \%$ & 2 & 6.00 & 7 \\
\hline $\begin{array}{l}\text { Young adult } \\
\text { women population }\end{array}$ & [170] & $\begin{array}{l}\text { Chlamydia Incidence and Re-infection } \\
\text { Rates Study (CIRIS) }\end{array}$ & 1116 & 21 & $79 \%$ & 3 & 1.00 & 13 \\
\hline Overall Mean (Std Dev) & & & 3459 (8979) & $24.7(23.5)$ & $\begin{array}{l}73.9 \% \\
(20.1 \%)\end{array}$ & $\begin{array}{l}4.6 \\
(8.0)\end{array}$ & $4.5(5.1)$ & $6.2(6.2)$ \\
\hline
\end{tabular}

retention $=70.1 \% ; 95 \% \mathrm{CI}[60.1 \%$ to $78.5 \%]$ ), after controlling for study duration and number of waves.

\section{Relationship between retention rate and retention strategy types}

A total of 95 retention strategies was identified, with an average of 6.2 strategies per article $(\mathrm{SD}=6.2)$. The most common retention strategies were: cash/voucher incentives to complete a follow-up assessment $(n=59)$, sending a postcard or letter reminder to complete a follow-up assessment $(n=43)$, and offering participants alternative methods of data collection, such as completing an interview face-to-face or over the phone $(n=36)$.

Retention strategies were grouped into four main retention strategy domains: (i) barrier-reduction strategies, such as offering childcare services, assistance with parking and transport, and engaging a participant sub-sample to evaluate data collection methods for the next wave; (ii) community-building strategies, such as creating a recognisable study brand via logos and colour schemes, giving away study merchandise to create a sense of project community (e.g., t-shirts with study logo), and sharing study results, news and events with participants via newsletters, social media, and feedback reports; (iii) strategies to improve follow-up rates within each wave, including cash or voucher incentives for varying levels of assessment completion, and use of phone calls, SMS, house visits, mail and email reminders to participants to complete assessments; and (iv) tracing strategies, such as collecting the details of alternative contact persons for each participant at baseline, using public or non-public records to find updated contact information for participants, and collecting detailed participant contact information via a locator document (e.g. full name, address, social security number, phone numbers, email addresses, etc.). The most commonly reported category was strategies to improve follow-up rates within waves, identified 306 times within the 143 cohorts, followed by barrier-reduction strategies (adopted 268 times), community-building strategies (adopted 181 times), and tracing strategies (adopted 138 times).

Table 2 presents the retention strategies used, grouped by retention strategy domain. It compares the retention rate for those studies that did, or did not utilise a specific retention strategy type or domain. Of the 95 
individual retention strategies examined, three demonstrated moderation of the retention rate. First, improved retention was associated with offering participants alternative methods of data collection (e.g., completing an interview face-to-face or over the phone) (median retention using strategy $=86.1 \%$; median retention not using strategy $=76.3 \% ; \mathrm{b}=0.24, p=.01$ ), and having participants complete a locator document at baseline (median retention using strategy $=90.9 \%$; median retention not using strategy $=78.1 \% ; b=0.49, p=0.02$ ). Finally, lower retention was associated with use of phone call reminders to participants to complete a follow-up wave (median retention using strategy $=72.7 \%$; median retention not using strategy $=80.6 \% ; b=0.25, p=.05)$. There was weak evidence against the null hypothesis of no moderation effect for a further three strategies. This included having consistent research team members (median retention using strategy $=87.3 \%$; median retention not using strategy $=$ $78.1 \%$; $\mathrm{b}=0.67, p=.09$ ); offering site and home visits for data collection (median retention using strategy $=$ 83.9\%; median retention not using strategy $=77.4 \%$; $b$ $=0.46, p=.07$ ); and sending participants thank you, birthday or holiday cards (median retention using strategy $=84.9 \%$; median retention not using strategy $=77.5 \% ; \mathrm{b}=0.50, p=.07)$. There was no evidence to support a moderated retention rate by any other specific retention strategy type.

To examine whether the specific strategy domains of barrier-reduction, community-building, follow-up/reminder, and tracing retention strategies were associated with retention rate, a binary variable was created for each domain that denoted whether a study did or did not utilise one or more specific strategy types within that domain. As shown in Table 2, after controlling for study duration and number of waves, studies that utilised any barrier-reduction strategy had higher retention rates than those that did not use a barrier strategy (median retention using barrier strategies $=81.1 \%$; median retention not using barrier strategies $=70.7 \% ; \mathrm{b}=0.61, p=.01$ ). Again after controlling for the study duration and number of waves, surprisingly, articles that reported use of at least one follow-up/reminder strategy had lower retention rates when compared to studies that did not utilise any follow-up/reminder (median retention using follow-up/reminder strategies $=76.4 \%$; median retention not using follow-up/reminder strategies $=86.1 \% ; b=-0.32, p<.01$ ). No relationships were found between retention rate and the use of any community-building or tracing retention strategies.

\section{Relationship between retention rate and number of strategies used}

To examine whether the cumulative number of retention strategies was associated with retention rate, we meta-regressed retention rate on to continuous variables representing the cumulative number of strategies used across strategy domains, and then within each domain separately. Greater number of retention strategies used (across all domains) was not associated with higher retention rate $(\mathrm{b}=0.02$; $95 \% \mathrm{CI}[-0.12$ to 0.05$], p=.21)$. When examined within each domain, controlling for study duration and number of waves, we found accumulation of barrier-reduction strategies was associated with higher retention ( $\mathrm{b}=0.12$; $95 \% \mathrm{CI}[0.02$ to 0.22$] ; p=.02)$. In separate meta-regressions, no relationships with retention were identified between number of community-building strategies $(b=-0.03 ; 95 \% \mathrm{CI}[-0.18$ to 0.11$] ; p=0.63)$, follow-up strategies $(b=-0.03 ; 95 \% \mathrm{CI}[-0.14$ to 0.09$]$; $p=0.65)$, or tracing strategies $(\mathrm{b}=0.10 ; 95 \% \mathrm{CI}[-0.07$ to 0.28$] ; p=.25$ ).

\section{Identifying strongest independent predictors of retention rate}

Three separate meta-regression models were estimated to examine strongest predictors of retention rate within strategy domains and types. Table 3-Model 1 shows that when examining retention strategy types as cumulative variables for each domain, barrier-reduction was independently associated with higher retention $(b=0.17$; $95 \% \mathrm{CI}$ [0.03 to 0.31$] ; p=.02$ ) and follow-up strategies was independently associated with lower retention $(b=-0.15$; 95\% CI [ -0.29 to -0.01$] ; p=.04$ ) beyond the effects of other retention strategy types. By contrast, Table 3-Model 2 demonstrates that when the retention rate was regressed on to all the binary indicator variables denoting whether the study did or did not utilise at least one strategy within that domain, only the use of follow-up/reminder strategies was independently associated with reduced retention rate $(\mathrm{b}=-0.83$; 95\% CI [- 1.4 to -0.27$] ; p<.01)$.

Finally, we investigated whether the associations between individual strategies and retention rate remained after controlling for other effective individual strategies in a single model (see Table 3 Model 3). A meta-regression model was created by entering only individual retention strategies that were associated with a retention rate at the $p<.10$ level (as discussed in [23, 24]). Six individual strategies were eligible: (i) offering alternative methods of data collection; (ii) consistency in the research staff; (iii) offering site and home visits; (iv) thank you and birthday cards; (v) phone call reminders; and (vi) the use of a locator form (i.e., alternate contacts). Offering participants alternative methods of data collection was associated with improved retention, whilst the use of phone call reminders was associated with reduced retention $(\mathrm{b}=0.59 ; 95 \% \mathrm{CI}[0.13$ to 1.05$] ; p=0.01$; $\mathrm{b}=-0.72 ; 95 \% \mathrm{CI}[-1.18$ to -0.25$] ; p<.01$, respectively). No associations were found between retention rates and the remaining four individual strategies. 
Table 2 Median meta-analytic retention rates for each retention strategy

\begin{tabular}{|c|c|c|c|c|c|c|c|}
\hline & \multicolumn{2}{|c|}{ Studies using strategy } & \multicolumn{2}{|c|}{ Studies not using strategy } & \multirow{2}{*}{$\begin{array}{l}\text { Absolute } \\
\text { Difference }\end{array}$} & \multirow[t]{2}{*}{$P$} & \multirow[t]{2}{*}{$1^{2}$} \\
\hline & $N$ & $\begin{array}{l}\text { Retention Rate } \\
\text { (Lower Cl - Upper Cl) }\end{array}$ & $N$ & $\begin{array}{l}\text { Retention Rate } \\
\text { (Lower Cl - Upper Cl) }\end{array}$ & & & \\
\hline Reducing barriers to participation (Any vs None) & 109 & $0.81(0.77-0.84)$ & 34 & $0.71(0.62-0.78)$ & 0.10 & $0.01^{*}$ & $99.87 \%$ \\
\hline $\begin{array}{l}\text { Adapt materials for mixed abilities (e.g., non-English speaking } \\
\text { participants) }\end{array}$ & 4 & $0.74(0.37-0.93)$ & 139 & $0.79(0.75-0.82)$ & -0.05 & 0.67 & $99.88 \%$ \\
\hline Adjust inclusion criteria & 1 & & & & & & na \\
\hline Adjust lab to be more home-like, less clinical & 2 & $0.81(0.77-0.84)$ & 141 & $0.79(0.75-0.82)$ & 0.02 & 0.84 & $99.89 \%$ \\
\hline Advisory group & 2 & $0.68(0.58-0.77)$ & 141 & $0.79(0.75-0.82)$ & -0.11 & 0.56 & $99.89 \%$ \\
\hline Alternative method of data collection & 36 & $0.86(0.78-0.92)$ & 107 & $0.76(0.72-0.8)$ & 0.10 & $0.01^{* *}$ & $99.88 \%$ \\
\hline Anonymity for participants & 1 & & & & & & na \\
\hline Assistance with postage costs & 5 & $0.88(0.73-0.95)$ & 138 & $0.79(0.75-0.82)$ & 0.09 & 0.21 & $99.89 \%$ \\
\hline Assistance with transport/parking/directions & 12 & $0.8(0.73-0.86)$ & 131 & $0.79(0.75-0.82)$ & 0.01 & 0.72 & $99.88 \%$ \\
\hline Catering/refreshments & 10 & $0.87(0.8-0.92)$ & 133 & $0.78(0.74-0.82)$ & 0.09 & 0.13 & $99.88 \%$ \\
\hline Child care & 3 & $0.68(0.51-0.82)$ & 140 & $0.79(0.75-0.82)$ & -0.11 & 0.36 & $99.89 \%$ \\
\hline Consistency in research staff & 11 & $0.87(0.77-0.93)$ & 132 & $0.78(0.74-0.82)$ & 0.09 & 0.09 & $99.88 \%$ \\
\hline Partial data collected from proxy/data linkage & 27 & $0.81(0.73-0.86)$ & 116 & $0.79(0.74-0.82)$ & 0.02 & 0.42 & $99.88 \%$ \\
\hline Adapt materials for different languages & 12 & $0.84(0.72-0.92)$ & 131 & $0.78(0.75-0.82)$ & 0.06 & 0.39 & $99.88 \%$ \\
\hline Extended data collection window & 7 & $0.74(0.54-0.88)$ & 136 & $0.79(0.75-0.82)$ & -0.05 & 0.52 & $99.88 \%$ \\
\hline Flexibility from research team (e.g., hours called, scheduling) & 24 & $0.83(0.76-0.89)$ & 119 & $0.78(0.74-0.82)$ & 0.05 & 0.23 & $99.88 \%$ \\
\hline Focus group on survey design & 2 & $0.72(0.7-0.75)$ & 141 & $0.79(0.75-0.82)$ & -0.07 & 0.93 & $99.89 \%$ \\
\hline Hiring, training, and support of staff & 21 & $0.84(0.77-0.9)$ & 122 & $0.78(0.74-0.82)$ & 0.06 & 0.11 & $99.88 \%$ \\
\hline $\begin{array}{l}\text { Matching staff to participants, e.g., by language spoken, } \\
\text { nature of questions }\end{array}$ & 2 & $0.94(0.91-0.96)$ & 141 & $0.79(0.75-0.82)$ & 0.15 & 0.14 & $99.88 \%$ \\
\hline Minimising time between data collection points & 1 & & & & & & na \\
\hline Pilot testing & 4 & $0.81(0.63-0.91)$ & 139 & $0.79(0.75-0.82)$ & 0.02 & 0.93 & $99.89 \%$ \\
\hline Prioritising measures & 12 & $0.73(0.6-0.82)$ & 131 & $0.8(0.76-0.83)$ & -0.07 & 0.37 & $99.89 \%$ \\
\hline Recruiting for long-term retention & 10 & $0.83(0.67-0.92)$ & 133 & $0.79(0.75-0.82)$ & 0.04 & 0.50 & $99.87 \%$ \\
\hline $\begin{array}{l}\text { Schedule two participants simultaneously - often family or } \\
\text { friends }\end{array}$ & 2 & $0.76(0.66-0.84)$ & 141 & $0.79(0.75-0.82)$ & -0.03 & 0.92 & $99.89 \%$ \\
\hline Simple, efficient procedure & 1 & & & & & & na \\
\hline Site and home visits & 31 & $0.84(0.78-0.88)$ & 112 & $0.77(0.73-0.81)$ & 0.07 & 0.07 & $99.88 \%$ \\
\hline Skip waves & 15 & $0.84(0.75-0.9)$ & 128 & $0.78(0.74-0.82)$ & 0.06 & 0.25 & $99.88 \%$ \\
\hline Splitting data collection over multiple sessions & 2 & $0.79(0.78-0.81)$ & 141 & $0.79(0.75-0.82)$ & 0.00 & 0.90 & $99.89 \%$ \\
\hline Survey design (e.g., order of survey items) & 3 & $0.77(0.52-0.91)$ & 140 & $0.79(0.75-0.82)$ & -0.02 & 0.88 & $99.88 \%$ \\
\hline Toll-free project phone number & 5 & $0.75(0.57-0.88)$ & 138 & $0.79(0.75-0.82)$ & -0.04 & 0.75 & $99.89 \%$ \\
\hline Creating a project community (Any vs None) & 59 & $0.80(0.75-0.85)$ & 84 & $0.78(0.73-0.82)$ & 0.02 & 0.48 & $99.88 \%$ \\
\hline Advisory group & 2 & $0.68(0.58-0.77)$ & 141 & $0.79(0.75-0.82)$ & -0.11 & 0.56 & $99.89 \%$ \\
\hline Branding & 14 & $0.79(0.65-0.89)$ & 129 & $0.79(0.75-0.82)$ & 0.00 & 0.99 & $99.88 \%$ \\
\hline Certificate of appreciation/completion & 2 & $0.83(0.28-0.98)$ & 141 & $0.79(0.75-0.82)$ & 0.04 & 0.82 & $99.88 \%$ \\
\hline Champion participants & 1 & & & & & & na \\
\hline Educating the community on research & 5 & $0.87(0.7-0.95)$ & 138 & $0.79(0.75-0.82)$ & 0.08 & 0.40 & $99.89 \%$ \\
\hline Emphasising benefits of study & 3 & $0.82(0.7-0.9)$ & 140 & $0.79(0.75-0.82)$ & 0.03 & 0.79 & $99.89 \%$ \\
\hline Events/opportunity to meet other participants & 9 & $0.69(0.54-0.82)$ & 134 & $0.8(0.76-0.83)$ & -0.11 & 0.23 & $99.88 \%$ \\
\hline Feedback report & 10 & $0.84(0.73-0.91)$ & 133 & $0.79(0.75-0.82)$ & 0.05 & 0.39 & $99.88 \%$ \\
\hline Gaining support of relevant institutions and organisations & 4 & $0.85(0.71-0.93)$ & 139 & $0.79(0.75-0.82)$ & 0.06 & 0.57 & $99.89 \%$ \\
\hline Gift/ freebies & 19 & $0.8(0.67-0.88)$ & 124 & $0.79(0.75-0.82)$ & 0.01 & 0.90 & $99.89 \%$ \\
\hline
\end{tabular}


Table 2 Median meta-analytic retention rates for each retention strategy (Continued)

\begin{tabular}{|c|c|c|c|c|c|c|c|}
\hline & \multicolumn{2}{|c|}{ Studies using strategy } & \multicolumn{2}{|c|}{$\underline{\text { Studies not using strategy }}$} & \multirow{2}{*}{$\begin{array}{l}\text { Absolute } \\
\text { Difference }\end{array}$} & \multirow[t]{2}{*}{$P$} & \multirow[t]{2}{*}{$1^{2}$} \\
\hline & N & $\begin{array}{l}\text { Retention Rate } \\
\text { (Lower Cl - Upper Cl) }\end{array}$ & N & $\begin{array}{l}\text { Retention Rate } \\
\text { (Lower Cl - Upper Cl) }\end{array}$ & & & \\
\hline Hiring, training, and support of staff & 21 & $0.84(0.77-0.9)$ & 122 & $0.78(0.74-0.82)$ & 0.06 & 0.11 & $99.88 \%$ \\
\hline Letter from chief investigator & 1 & & & & & & na \\
\hline Media coverage & 3 & $0.7(0.69-0.72)$ & 140 & $0.79(0.75-0.82)$ & -0.09 & 0.82 & $99.89 \%$ \\
\hline Newsletter/e-newsletter & 24 & $0.83(0.76-0.89)$ & 119 & $0.78(0.74-0.82)$ & 0.05 & 0.23 & $99.88 \%$ \\
\hline Opportunity to participate in other research & 1 & & & & & & na \\
\hline Photo album & 2 & $0.72(0.69-0.75)$ & 141 & $0.79(0.75-0.82)$ & -0.07 & 0.75 & $99.89 \%$ \\
\hline Building rapport & 22 & $0.79(0.69-0.86)$ & 121 & $0.79(0.75-0.82)$ & 0.00 & 0.97 & $99.89 \%$ \\
\hline Sharing study results & 5 & $0.88(0.66-0.97)$ & 138 & $0.79(0.75-0.82)$ & 0.09 & 0.24 & $99.89 \%$ \\
\hline Social media & 2 & $0.89(0.72-0.96)$ & 141 & $0.79(0.75-0.82)$ & 0.10 & 0.39 & $99.89 \%$ \\
\hline Study membership card & 1 & & & & & & na \\
\hline Thank you, birthday, and holiday cards & 25 & $0.85(0.79-0.9)$ & 118 & $0.78(0.73-0.81)$ & 0.07 & 0.07 & $99.88 \%$ \\
\hline Time with chief investigator & 2 & $0.92(0.8-0.97)$ & 141 & $0.79(0.75-0.82)$ & 0.13 & 0.24 & $99.89 \%$ \\
\hline Website & 3 & $0.80(0.47-0.94)$ & 140 & $0.79(0.75-0.82)$ & 0.01 & 1.00 & $99.88 \%$ \\
\hline Follow-up/Reminder strategies (Any vs None) & 111 & $0.76(0.72-0.80)$ & 32 & $0.86(0.79-0.91)$ & -0.10 & $0.02^{*}$ & $99.86 \%$ \\
\hline Follow-up brochure & 2 & $0.78(0.74-0.81)$ & 141 & $0.79(0.75-0.82)$ & -0.01 & 0.97 & $99.89 \%$ \\
\hline Budgeting for multiple contact attempts & 1 & & & & & & na \\
\hline Extra incentive to complete all data collection points & 2 & $0.93(0.77-0.98)$ & 141 & $0.79(0.75-0.82)$ & 0.14 & 0.17 & $99.89 \%$ \\
\hline Gift/ freebies incentives (e.g., t-shirts, discount cards) & 18 & $0.8(0.67-0.88)$ & 125 & $0.79(0.75-0.82)$ & 0.01 & 0.90 & $99.89 \%$ \\
\hline Hiring, training, and support of staff & 21 & $0.84(0.77-0.9)$ & 122 & $0.78(0.74-0.82)$ & 0.06 & 0.11 & $99.88 \%$ \\
\hline Incentive (cash/vouchers) & 59 & $0.78(0.72-0.82)$ & 84 & $0.8(0.75-0.84)$ & -0.02 & 0.45 & $99.88 \%$ \\
\hline Incentive increasing value over time & 10 & $0.78(0.62-0.88)$ & 133 & $0.79(0.75-0.82)$ & -0.01 & 0.81 & $99.88 \%$ \\
\hline Incentives raffles/competitions & 11 & $0.86(0.71-0.94)$ & 132 & $0.78(0.75-0.82)$ & 0.08 & 0.22 & $99.88 \%$ \\
\hline Increased incentive for hard-to-reach Pp & 6 & $0.68(0.47-0.84)$ & 137 & $0.79(0.76-0.83)$ & -0.11 & 0.24 & $99.88 \%$ \\
\hline Limiting number of calls etc. based on participants' response & 1 & & & & & & na \\
\hline Medical assistance (e.g., diagnostic testing) & 27 & $0.74(0.64-0.82)$ & 116 & $0.8(0.76-0.84)$ & -0.06 & 0.17 & $99.88 \%$ \\
\hline Phone Follow-up & 11 & $0.80(0.67-0.89)$ & 132 & $0.79(0.75-0.82)$ & 0.01 & 0.90 & $99.88 \%$ \\
\hline Provide referrals, e.g., medical or legal & 9 & $0.85(0.77-0.91)$ & 134 & $0.78(0.75-0.82)$ & 0.07 & 0.26 & $99.89 \%$ \\
\hline Resend survey once & 6 & $0.77(0.64-0.86)$ & 137 & $0.79(0.75-0.82)$ & -0.02 & 0.79 & $99.88 \%$ \\
\hline Resend survey multiple times & 10 & $0.76(0.64-0.84)$ & 133 & $0.79(0.75-0.83)$ & -0.03 & 0.63 & $99.88 \%$ \\
\hline SMS follow-up & 1 & & & & & & na \\
\hline Website follow-up & 8 & $0.81(0.62-0.91)$ & 135 & $0.79(0.75-0.82)$ & 0.02 & 0.93 & $99.88 \%$ \\
\hline Email reminder & 13 & $0.73(0.58-0.85)$ & 130 & $0.79(0.76-0.83)$ & -0.06 & 0.31 & $99.88 \%$ \\
\hline Face-to-face reminder (e.g., home visit) & 7 & $0.85(0.67-0.94)$ & 136 & $0.79(0.75-0.82)$ & 0.06 & 0.33 & $99.89 \%$ \\
\hline Phone call reminder & 34 & $0.73(0.63-0.8)$ & 109 & $0.81(0.77-0.84)$ & -0.08 & $0.05^{*}$ & $99.88 \%$ \\
\hline Postcard/letter reminder & 43 & $0.77(0.7-0.83)$ & 100 & $0.80(0.75-0.84)$ & -0.03 & 0.50 & $99.88 \%$ \\
\hline SMS reminder & 5 & $0.85(0.8-0.9)$ & 138 & $0.79(0.75-0.82)$ & 0.06 & 0.42 & $99.89 \%$ \\
\hline Reminders (unspecified) & 1 & & & & & & na \\
\hline Tracing strategies (Any vs None) & 53 & $0.80(0.73-0.85)$ & 90 & $0.78(0.74-0.83)$ & 0.02 & 0.62 & $99.88 \%$ \\
\hline Tracing via alternative contacts & 28 & $0.82(0.75-0.87)$ & 115 & $0.78(0.74-0.82)$ & 0.04 & 0.32 & $99.88 \%$ \\
\hline Case-review meetings & 1 & & & & & & na \\
\hline Tracing via change of address cards & 2 & $0.74(0.43-0.91)$ & 141 & $0.79(0.75-0.82)$ & -0.05 & 0.95 & $99.89 \%$ \\
\hline Tracing via email & 2 & $0.74(0.43-0.92)$ & 141 & $0.79(0.75-0.82)$ & -0.05 & 0.82 & $99.89 \%$ \\
\hline
\end{tabular}


Table 2 Median meta-analytic retention rates for each retention strategy (Continued)

\begin{tabular}{|c|c|c|c|c|c|c|c|}
\hline & \multicolumn{2}{|c|}{ Studies using strategy } & \multicolumn{2}{|c|}{ Studies not using strategy } & \multirow{2}{*}{$\begin{array}{l}\text { Absolute } \\
\text { Difference }\end{array}$} & \multirow[t]{2}{*}{$P$} & \multirow[t]{2}{*}{$1^{2}$} \\
\hline & $N$ & $\begin{array}{l}\text { Retention Rate } \\
\text { (Lower Cl - Upper Cl) }\end{array}$ & N & $\begin{array}{l}\text { Retention Rate } \\
\text { (Lower Cl - Upper Cl) }\end{array}$ & & & \\
\hline $\begin{array}{l}\text { Extensive location tracking information, e.g., known } \\
\text { 'hangouts' }\end{array}$ & 1 & & & & & & na \\
\hline Hiring, training, and support of staff & 21 & $0.84(0.77-0.9)$ & 122 & $0.78(0.74-0.82)$ & 0.06 & 0.11 & $99.88 \%$ \\
\hline Tracing via house visit & 1 & & & & & & na \\
\hline Tracing via incentive for staff members & 2 & $0.72(0.67-0.76)$ & 141 & $0.79(0.75-0.82)$ & -0.07 & 0.69 & $99.89 \%$ \\
\hline Tracing via incentive to update contact details & 3 & $0.86(0.62-0.96)$ & 140 & $0.79(0.75-0.82)$ & 0.07 & 0.43 & $99.88 \%$ \\
\hline Tracing via letter & 9 & $0.77(0.51-0.91)$ & 134 & $0.79(0.75-0.82)$ & -0.02 & 0.72 & $99.89 \%$ \\
\hline Tracing via locator form documentation* & 7 & $0.91(0.79-0.97)$ & 136 & $0.78(0.74-0.81)$ & 0.13 & $0.02^{*}$ & $99.88 \%$ \\
\hline Tracing via phone call & 8 & $0.67(0.51-0.8)$ & 135 & $0.8(0.76-0.83)$ & -0.13 & 0.12 & $99.88 \%$ \\
\hline Tracing via private investigator & 1 & & & & & & na \\
\hline Tracing via SMS & 1 & & & & & & na \\
\hline Tracing via social media & 3 & $0.79(0.39-0.95)$ & 140 & $0.79(0.75-0.82)$ & 0.00 & 0.92 & $99.89 \%$ \\
\hline Tracing via tracing via public records & 20 & $0.82(0.73-0.88)$ & 123 & $0.78(0.74-0.82)$ & 0.04 & 0.37 & $99.88 \%$ \\
\hline Tracing via tracking database & 15 & $0.83(0.73-0.9)$ & 128 & $0.78(0.74-0.82)$ & 0.05 & 0.32 & $99.88 \%$ \\
\hline Tracing via update your details form & 4 & $0.9(0.81-0.96)$ & 139 & $0.79(0.75-0.82)$ & 0.11 & 0.15 & $99.89 \%$ \\
\hline Tracing via website & 2 & $0.80(0.79-0.81)$ & 141 & $0.79(0.75-0.82)$ & 0.01 & 0.99 & $99.89 \%$ \\
\hline $\begin{array}{l}\text { Tracing via non-public records, e.g., apartment complex } \\
\text { managers }\end{array}$ & 7 & $0.82(0.66-0.92)$ & 136 & $0.79(0.75-0.82)$ & 0.03 & 0.59 & $99.89 \%$ \\
\hline
\end{tabular}

All inferential analyses adjusted for study duration and number of waves

na insufficient studies to perform meta-analysis

$N$ No. effect in analysis

${ }^{*} p<.05$

${ }^{* *} p<.01$

Relationship between retention rate and emerging strategies The final group-level analysis investigated the association between emerging retention strategies and retention rates. Within these 95 retention strategies, 44 emerging strategies were identified, including the application of social media and SMS to assist in tracing participants lost to follow-up, and the application of study websites and social media profiles for keeping participants up-to-date with the study's news and events. Meta-regressions demonstrated that articles reporting a higher frequency of emerging retention strategies had higher retention, after controlling for study duration and number of waves $(b=0.08 ; 95 \%$ CI $[0.01$ to 0.16$] ; p=.03)$. Despite this, there was no difference in overall retention rates between those articles that did and did not report the use of emerging retention strategies (median retention using emerging strategies $=80.1 \%$; median retention not using emerging strategies $=75.0 \% ; \mathrm{b}=0.27, p=.27)$.

\section{Discussion}

This study aimed to identify retention strategies employed in longitudinal cohort studies during the past decade, and to examine their effectiveness. We identified 143 longitudinal cohort studies that described retention strategies and outcomes, resulting in 95 different retention strategies. We then investigated whether study or participant characteristics moderated retention, the relationship between retention rate and retention strategy type, and whether new cohort retention strategies have emerged since previous reviews. In so doing, this study is the first meta-analysis of retention strategies conducted in longitudinal cohort studies. This research particularly complements the previous narrative review that investigated cohort retention strategies in longitudinal research [6], and the wider literature investigating participant retention strategies across health research designs (e.g., 4,16,17). Such research has important implications for maximising cohort retention and reducing research administration costs, which will subsequently improve the efficacy and quality of health research.

We first investigated how study or participant characteristics may influence cohort retention. Study characteristics included sample size, study duration, number of waves, and country development level - none of which were associated with retention rate. Participant characteristics included mean age at baseline, cohort type (clinical or non-clinical), and gender. We found that cohort studies with a higher proportion of male participants had lower retention rates than studies with a higher 
Table 3 Meta-analytic regression results between retention strategy themes and retention rate

\begin{tabular}{|c|c|c|c|c|}
\hline & Estimate & $\mathrm{Cl}$ (Lower - Upper) & $P$ & $1^{2}$ \\
\hline Model 1: Continuous total number of retention strategy types & & & & $99.86 \%$ \\
\hline Barriers & 0.17 & $0.03-0.32$ & $0.02^{*}$ & \\
\hline Community & -0.03 & $-0.18-0.11$ & 0.63 & \\
\hline Follow-up/reminder & -0.15 & $-0.29--0.01$ & $0.04^{*}$ & \\
\hline Tracing & 0.11 & $-0.06-0.27$ & 0.22 & \\
\hline Study duration & -0.04 & $-0.08-0.00$ & 0.06 & \\
\hline Number of waves & 0.00 & $-0.02-0.03$ & 0.81 & \\
\hline Model 2: Binary usage of retention strategy types & & & & $99.84 \%$ \\
\hline Barriers & 0.35 & $-0.15-0.86$ & 0.16 & \\
\hline Community & 0.35 & $-0.14-0.83$ & 0.16 & \\
\hline Follow-up/reminder & -0.83 & $-1.40--0.27$ & $0.00^{* *}$ & \\
\hline Tracing & 0.11 & $-0.36-0.59$ & 0.64 & \\
\hline Study duration & -0.03 & $-0.08-0.01$ & 0.10 & \\
\hline Number of waves & 0.01 & $-0.02-0.03$ & 0.61 & \\
\hline Model 3: All individual strategies with $p<0.1$ & & & & $99.85 \%$ \\
\hline Tracing - Locator form documentation & 0.59 & $-0.44-1.62$ & 0.26 & \\
\hline Follow-up - Reminder Phone call & -0.72 & $-1.20--0.25$ & $0.00^{* *}$ & \\
\hline Community - Thank you and birthday cards & 0.44 & $-0.11-0.98$ & 0.12 & \\
\hline Barriers - Site and home visits & 0.42 & $-0.05-0.88$ & 0.08 & \\
\hline Barriers - Consistency in research staff & 0.39 & $-0.42-1.20$ & 0.34 & \\
\hline Barriers - Alternative method of data collection & 0.59 & $0.14-1.05$ & $0.01^{* *}$ & \\
\hline Study duration & -0.04 & $-0.08--0.00$ & $0.05^{*}$ & \\
\hline Number of waves & -0.00 & $-0.03-0.02$ & 0.89 & \\
\hline
\end{tabular}

proportion of female participants; no associations were found for participants' age or cohort type. While difficulties in retaining male participants are well-documented in previous research (e.g., 4,20,21), our study noted that cohorts with a higher proportion of male participants were also more likely to be clinical samples than cohorts with a higher proportion of female participants. In addition, cohorts with a higher proportion of male participants were also disproportionately focused on high-risk groups, such as substance use and men who have sex with men (e.g., the Bangkok Men who have Sex with Men Cohort Study (BMCS) [25] and the International Multicenter ADHD Genetics (IMAGE) study [26]). Thus, the difficulties in retention reported in this study and the wider literature could potentially be attributed to the differential impact of these clinical issues that affect men more than women. Researchers working with hard-to-retain populations, such as men in particular clinical groupings, may benefit from investigating what retention strategies work within their specific populations and settings beyond the core retention strategies identified in this review.
Second, we investigated the relationship between retention rate and retention strategies. We identified 95 different retention strategies, grouped thematically into four classes: barrier-reduction, community-building, follow-up, and tracing. Specific strategies associated with improved retention rates included the barrier-reduction strategy of offering alternative methods of data collection to participants (e.g., completing an interview over the phone or in person); and the tracing strategy of collecting detailed contact information from participants at baseline via a locator document. Further, weak evidence was found for one community-building and two further barrier-reduction strategies: (i) sending participants thank you, birthday or holiday cards; (ii) having consistent research team members, and; (iii) offering site and home visits for data collection.

Overall, barrier-reduction strategies emerged as the strongest predictor of improved retention. Barrier-reduction strategies may be particularly useful in longitudinal research given participants are likely to experience significant changes in their capacity to remain involved across the study's duration (typically years). Follow-up/reminder 
strategies, such as incentives and reminders, were associated with significantly poorer retention. This result was surprising, given that the previous review investigating retention strategies in longitudinal cohort studies found the opposite, that use of these follow-up/reminder strategies resulted in improved retention rates [6]. The lack of support for follow-up/reminder strategies found in the current review could be due to a number of extraneous variables including: (i) timing: studies may have implemented this strategy after other retention efforts proved ineffective; (ii) participant burden: the studies using follow-up/reminder strategies may have involved a high data collection burden (e.g., long surveys); (iii) sampling: studies using follow-up/reminder strategies may be over-represented in studies of difficult-to-retain populations, such as men. However, these explanations are unlikely, given that follow-up/reminder strategies were identified in most of the cohorts included in this review (111 out of the 143 cohorts), and the cohorts employing follow-up/reminder strategies did not differ by research design (sample size: $t_{(141)}=.67, p=.50$; no. waves: $t_{(141)}=$ -.43, $p=.67$ ) or participant characteristics (age: $t_{(141)}=$ -.11, $p=.91$; gender: $\chi_{(2, n=143)}^{2}=.37, p=.85$; HDI: $\chi^{2}(2, \mathrm{n}$ $=143)=.01, p=.97)$. Differences were observed only for study duration (any $M(\mathrm{SD})=3.9(4.4)$; none $(\mathrm{SD})=5.8(6.4)$; $\left.t_{(141)}=2.00, p=.05\right)$. Alternatively, participants may perhaps view follow-up/reminder strategies as the research team "badgering" them to complete assessments, thereby damaging rapport. This negative perspective of follow-up/reminder strategies may be further exacerbated if the research team has not implemented sufficient barrier-reduction strategies to help make it easier for participants to remain involved in the study. Future research could consider investigating participants' perspectives of retention strategies in longitudinal cohort studies, ensuring that both active and inactive participants are included, to better understand the costs and benefits of different approaches.

Interestingly, the current study found that simply adding more cohort retention strategies did not result in higher retention rates. These results contradict the findings of Robinson et al. [17] and Davis et al. [4], who both found that the use of more retention strategies across multiple classes was associated with improved retention rates. However, neither study specifically examined participant retention in longitudinal cohort studies, and both synthesised their retention results using a narrative rather than meta-analytic approach. Given that the implementation of retention strategies can be costly in terms of both time and money, the overall number of strategies employed is important to evaluate. The interaction of quantity of retention strategies used and provision of flexibility needs to be better understood, given research protocols that accommodate the changing lives of participants should remain a key focus of retention efforts.
Finally, we examined whether studies utilising new or emerging retention strategies had improved retention compared with studies using established strategies. Of the 95 retention strategies described in the included articles, 44 were identified as an emerging retention strategy that had not yet been described in extant systematic reviews examining participant retention $[4,6,16,17]$. Emerging strategies included using social media and SMS to assist in tracing participants lost to follow-up, and the use of study websites and social media profiles for keeping participants up-to-date with study news and events. Emerging retention strategies were endorsed by only a handful of studies, and the use of a single emerging strategy was not significantly associated with retention rate. However, we found that studies that employed more emerging retention strategies were associated with improved retention rates. Importantly, emerging strategies were identified across all four retention strategy domains (barrier-reduction, community-building, follow-up/reminder, and tracing), demonstrating that the association between emerging strategies and improvements in retention are due to the use of modern technology to help achieve core cohort engagement goals. Thus, we recommend that researchers continue to innovate their retention efforts, particularly where such strategies may reduce participant burden.

The current study has a number of limitations. First, the number of articles that focused on reporting retention strategies in detail was proportionally low compared to the number of articles that did not focus on reporting retention strategies. Although retention strategies were identified within 143 longitudinal cohorts, only 55 included cohort retention as a key focus area. Very few articles $(n=12)$ were identified that reported strategy-specific retention rates within the longitudinal cohort studies. The number of retention strategies reported by articles ranged from one to 32, with 35 of the 141 articles describing only one retention strategy. Longitudinal cohort studies should aim to publish protocol papers that delineate their cohort retention strategies, and ensure that the protocol is updated as retention efforts evolve.

Second, net retention rates were calculated by the difference between the first and last wave of data collection reported in the article. Where specified, ineligible participants (e.g., participants recruited after the first wave, or deceased participants) were excluded from the retention rate calculation. However, some articles did not provide detailed information on the eligibility of the sample at the final wave, and thus it is possible that the retention rates calculated for some studies may be slightly inaccurate. This limitation could be addressed by researchers providing details on the eligibility of their samples at each wave.

Third, high levels of heterogeneity were reported for most analyses in this study. This may best be explained 
by two factors. First, we expected to identify high heterogeneity given the diversity of research questions, methodologies, and cohorts reported across articles. Second, only a small number of studies were eligible for most meta-regressions in this paper, which reduces the precision of heterogeneity estimates [27]. This limitation could be addressed in future work, which could aim to investigate the effectiveness of different retention strategies within different subgroups.

Finally, by nature of synthesising retention results across different samples and settings, the current study is unable to disaggregate nuanced effects of various retention strategies across specific contexts and populations, given results are pooled across multiple studies. The current study did address this broadly by investigating the effects of study and sample characteristics on retention.

A final point to note is that available to researchers are a range of statistical or methodological approaches that can minimise potential biases introduced with attrition. Whilst beyond the scope of this paper, these approaches include formal statistical methods for addressing missingness due to attrition such as multiple imputation or full information maximum likelihood methods [28, 29]. Moreover, researchers may address attrition methodologically by using replacement sampling approaches that recruit new participants into a study to replace those who have dropped out, based on shared characteristics measured in the original sampling frame [30, 31]. All these methods provide useful avenues to address attrition once any employed retention strategies have been used to retain the largest proportion of the original sample as possible.

\section{Conclusions}

Overall, this study has important implications for the retention efforts of longitudinal cohort studies. Combined, these results suggest that researchers need to be strategic in choosing how to invest their resources to better target participant retention, rather than simply increasing the number of strategies applied. Projects should invest both time and funding into matching retention strategies to the sample prior to implementation, including careful consideration of unintended burden for participants. Finally, given the high number of emerging retention strategies identified, longitudinal research methods clearly continue to evolve. Longitudinal cohort studies may benefit from open and regular protocol revision to incorporate new strategies, particularly where these strategies may offer greater flexibility to participants.

\section{Additional file}

Additional file 1: Table S1. Terms used in the electronic search strategy, adjusted as required for each database. (DOCX 12 kb)

\section{Abbreviations}

ADHD: Attention Deficit Hyperactivity Disorder; AlBL: Australian Imaging, Biomarkers and Lifestyle Flagship Study of Ageing; AIDS: Acquired Immune Deficiency Syndrome; ATAP: Australian Twin ADHD project; BGALS: Berkeley Girls with ADHD Longitudinal Study; BMCS: Bangkok Men who have Sex with Men Cohort Study; BT20: Birth to Twenty birth cohort; CAS(K): Knee clinical assessment study; CHS: Cardiovascular health study; Cl: Confidence interval; CIRIS: Chlamydia Incidence and Re-infection Rates Study; CLHLS: Chinese Longitudinal Healthy Longevity Survey; COHRA2: Center for Oral Health Research in Appalachia 2 Study; DCHS: Drakenstein Child Health Study; EARTH: Education and Research Towards Health study; ECAGE: Study of Food Intake and Eating Behavior of Pregnant Women; EHL: Environments for Healthy Living; FAMAS: Florey Adelaide Male Ageing Study;

FIOCRUZ: Fundação Oswaldo Cruz Rio de Janeiro; GGNET: G-GrippeNet Project; GROW: Geographic research on wellbeing study; HANDLS: Healthy Aging in Neighborhoods of Diversity across the Life Span study; HAS: Helsinki Aging Study; HDI: Human Development Index; Heart SCORE: Heart Strategies Concentrating on Risk Evaluation study; HIV: Human Immunodeficiency Virus infection; IMAGE: International Multicenter ADHD Genetics study; IMPAACT: International Maternal Pediatric Adolescent AIDS Clinical Trials; IPEC: Instituto de Pesquisa Clínica Evandro Chagas; IYDS: International Youth Development Study; JCS: Japan Children's Study; LASA: Longitudinal Aging Study Amsterdam; LAW: Longitudinal Assessment of Women; M: Mean; MARI: Maternal Anxiety in Relation to Infant Development Study; MASALA: Mediators of Atherosclerosis in South Asians Living in America study; MIDUS: Midlife in the United States; MLS: Maternal Lifestyle Study; MTFS: Minnesota Twin Family Study; MUSP: Mater-University Study of Pregnancy; NECS: New England Centenarian Study; NEMESIS-

2: Netherlands Mental Health Survey and Incidence Study;

NESDA: Netherlands Study of Depression and Anxiety; NINFEA: Nascita e INFanzia gli Effetti dell'Ambiente cohort; NLLFS: US National Longitudinal Lesbian Family Study; NSHD: National Survey of Health and Development; OPPERA: Orofacial Pain: Prospective Evaluation and Risk Assessment Study; PRC: People's Republic of China; PRISMA: Preferred Reporting Items for Systematic Reviews and Meta-Analyses; QLS: Quinte Longitudinal Study; RHIAA: Religion and Health in African Americans study; SD: Standard Deviation; SELF: Study of Environment, Lifestyle and Fibroids; SHIP: Study of Health in Pomerania; SMS: Short Message Services; SUPERB: Study of Use of Products and Exposure-Related Behavior; TEDDY: The Environmental Determinants of Diabetets in the Young study; TNTR: Tasmanian Neurotrauma Register; TRAILS: Tracking Adolescents' Individual Lives Survey; UAB Study of Ageing: University of Alabama at Birmingham Study of Aging; UFS: Uterine Fibroid Study; USA: United States of America; USC RFAB: University of Southern California Study of Risk Factors for Antisocial Behavior; WCLS: Welfare Client Longitudinal Study; YAS: Youth Asset Study

\section{Acknowledgements}

This paper represents a collaborative effort on behalf of the Centre for Social and Early Emotional Development (SEED) Lifecourse Sciences Theme. The contribution from authors to this paper was part of a broader collaborative effort from SEED Lifecourse Sciences Theme members. We request that investigators who are members of the theme be listed at the end of the manuscript. Contributions made by other individuals have been acknowledged in the usual way.

^SEED Lifecourse Sciences Theme: Including the primary investigators already listed and: Sharyn Bant, Sophie Barker, Anna Booth, Tanja Capic, Laura Di Manno, Alisha Gulenc, Genevieve Le Bas, Primrose Letcher, Claire Ann Lubotzky, Gypsy O'Dea, Jessica Opie, Melissa O'Shea, Evelyn Tan, and Jo Williams.

\section{Funding}

This work was supported by the Centre for Social and Early Emotional Development (SEED), Faculty of Health, Deakin University Geelong. Openaccess publishing costs for this work were supported by a Deakin University Faculty of Health HDR Open-Access Publishing Grant. DH is financially supported by an Australian Unity Senior Research Fellowship; and CO is financially supported by an Australian Research Council Principal Research Fellowship. 


\section{Availability of data and materials}

The datasets used and/or analysed during the current study are available from the corresponding author on reasonable request.

\begin{abstract}
Authors' contributions
ST conceptualised and designed the study, completed the data collection, carried out the analyses, contributed to the interpretation of the data, and wrote the manuscript. GY carried out the analyses, and contributed to the interpretation of the data, the write-up of the results and preparation of tables, and revision of the manuscript. JAM contributed to the data analysis, interpretation of the results, and the write-up and revision of the manuscript. ES conceptualised and designed the study, supervised data collection, and contributed to the interpretation of the data and revision of the manuscript. AS contributed to the data collection, interpretation of the results, and revision of the manuscript. MF contributed to the data analysis, interpretation of the results and revision of the manuscript. CG, JM, and CO critically reviewed and revised the manuscript for important intellectual content. DH conceptualised and designed the study, coordinated and supervised data collection and the preparation of data for analysis, and contributed to the interpretation of the data and revision of the manuscript. All authors approved the final manuscript as submitted and agree to be accountable for all aspects of the work.
\end{abstract}

\section{Ethics approval and consent to participate}

Not applicable.

\section{Consent for publication}

Not applicable.

\section{Competing interests}

The authors declare that they have no competing interests.

\section{Publisher's Note}

Springer Nature remains neutral with regard to jurisdictional claims in published maps and institutional affiliations.

\section{Author details \\ 'Centre for Social and Early Emotional Development, School of Psychology, Faculty of Health, Deakin University, Burwood, Geelong, Victoria 3125, Australia. ${ }^{2}$ Murdoch Children's Research Institute, Centre for Adolescent Health, Royal Children's Hospital, Melbourne, Australia. ${ }^{3}$ Department of Paediatrics, Royal Children's Hospital, University of Melbourne, Melbourne, Australia. ${ }^{4}$ Melbourne School of Psychological Sciences, University of Melbourne, Parkville, Australia. ${ }^{5}$ National Drug and Alcohol Research Centre, University of New South Wales, Sydney, Australia. ${ }^{6}$ School of Engineering \& Information Technology, Faculty of Science \& Technology, Federation University, Melbourne, Australia.}

Received: 24 January 2018 Accepted: 23 October 2018 Published online: 26 November 2018

\section{References}

1. Rothman KJ, Greenland S. Cohort studies. In: Rothman KJ, Greenland S, Lash $T L$, editors. Modern epidemiology. Philadelphia: Lippincott Williams \& Wilkins; 2008.

2. Rothman K, Greenland S, Lash TL. Modern epidemiology. 3rd ed. Philadelphia: Lippincott Williams \& Wilkins; 2008.

3. Gustavson K, von Soest T, Karevold E, Røysamb E. Attrition and generalizability in longitudinal studies: findings from a 15-year populationbased study and a Monte Carlo simulation study. BMC Public Health. 2012; 12:918.

4. Davis L, Broome ME, Cox RP. Maximizing retention in community-based clinical trials. J Nurs Scholarsh. 2002;1:47-53.

5. Szklo M. Population-based cohort studies. Epidemiol Rev. 1998;20:81-90.

6. Booker CL, Harding S, Benzeval M. A systematic review of the effect of retention methods in population-based cohort studies. BMC Public Health 2011;11:249

7. Anderson CL, Agarwal R, Anderson CL. The digitization of healthcare: boundary risks, emotion, and consumer willingness to disclose personal health information. Inf Syst Res. 2011;22:469-90.

8. Ward S, Bridges K, Chitty B. Do incentives matter? An examination of on-line privacy concerns and willingness to provide personal and financial information. J Mark Commun. 2005;11:21-40.
9. Sepah SC, Jiang L, Peters AL. Long-term outcomes of a web-based diabetes prevention program: 2-year results of a single-arm longitudinal study. J Med Internet Res. 2015;17:e92.

10. Dobkin $\mathrm{BH}$, Dorsch $\mathrm{A}$. The promise of mHealth: daily activity monitoring and outcome assessments by wearable sensors. Neurorehabil Neural Repair. 2011;25:788-98.

11. Fuller-Tyszkiewicz M, Skouteris H, Richardson B, Blore J, Holmes M, Mills J. Does the burden of the experience sampling method undermine data quality in state body image research? Body Image. 2013;10:607-13.

12. Rostosky SS, Riggle EDB, Rothblum ED, Balsam KF. Same-sex couples' decisions and experiences of marriage in the context of minority stress: interviews from a population-based longitudinal study. J Homosex. 2016;63: $1019-40$.

13. Loxton D, Powers J, Anderson AE, Townsend N, Harris ML, Tuckerman R, et al. Online and offline recruitment of young women for a longitudinal health survey: findings from the australian longitudinal study on women's health 1989-95 cohort. J Med Internet Res. 2015;17:1-14.

14. Mychasiuk R, Benzies K. Facebook: an effective tool for participant retention in longitudinal research. Child Care Health Dev. 2012;38:753-6.

15. Man MS, Tilbrook HE, Jayakody S, Hewitt CE, Cox H, Cross B, et al. Electronic reminders did not improve postal questionnaire response rates or response times: a randomized controlled trial. J Clin Epidemiol. 2011;64:1001-4.

16. Brueton VC, Tierney JF, Stenning S, Meredith S, Harding S, Nazareth I, et al. Strategies to improve retention in randomised trials: a Cochrane systematic review and meta-analysis. BMJ Open. 2014;4:e003821.

17. Robinson KA, Dennison CR, Wayman DM, Pronovost PJ, Needham DM. Systematic review identifies number of strategies important for retaining study participants. J Clin Epidemiol. 2007;60:757-65.

18. Moher D, Liberati A, Tetzlaff J, Altman DG. Preferred reporting items for systematic reviews and meta analyses: the PRISMA statement. Ann Intern Med. 2009;151:264-9.

19. Viechtbauer W. Conducting Meta-analyses in R with the metafor package. J Stat Softw. 2010;36:1-48.

20. R Core Team. R: A language and environment for statistical computing. Vienna: R Foundation for Statistical Computing; 2015.

21. Higgins JPT, Thompson SG, Deeks JJ, Altman DG. Measuring inconsistency in meta-analyses. BMJ Br Med J. 2003;327:557-60.

22. United Nations Development Programme (UNDP). Human development report. New York: United Nations Development Programme; 2016.

23. Victora CG, Huttly SR, Fuchs SC, Olinto MT. The role of conceptual frameworks in epidemiological analysis: a hierarchical approach. Int J Epidemiol. 1997;26:224-7.

24. Hosmer J, Lemeshow S, Sturdivant R. Model-building strategies and methods for logistic regression. In: Applied logistic regression. 3rd ed. Hoboken: Wiley; 2013. p. 89-151.

25. Pattanasin S, Wimonsate W, Chonwattana W, Tongtoyai J, Chaikummao S, Sriporn A, et al. Loss to follow-up and bias assessment among a cohort of Thai men who have sex with men in Bangkok. Thailand Int J STD AIDS. 2016;27:196-206.

26. van Lieshout M, Luman M, Twisk JWR, van Ewijk H, Groenman AP, Thissen AJAM, et al. A 6-year follow-up of a large European cohort of children with attentiondeficit/hyperactivity disorder-combined subtype: outcomes in late adolescence and young adulthood. Eur Child Adolesc Psychiatry. 2016;25:1007-17.

27. Von Hippel PT. The heterogeneity statistic 12 can be biased in small metaanalyses. BMC Med Res Methodol. 2015;15:1-8.

28. Enders CK. Applied missing data analysis. New York: Guilford Press; 2010.

29. Graham JW. Missing data analysis: making it work in the real world. Annu Rev Psychol. 2009;60:549-76.

30. Vehovar V. Field substitution and unit nonresponse. J Off Stat. 1999; 15:335-50.

31. David MC, Bensink M, Higashi H, Donald M, Alati R, Ware RS. Monte Carlo simulation of the cost-effectiveness of sample size maintenance programs revealed the need to consider substitution sampling. J Clin Epidemiol. 2012; 65:1200-11.

32. Cepeda A, Valdez A. Ethnographic strategies in the tracking and retention of street-recruited community-based samples of substance using hidden populations in longitudinal studies. Subst Use Misuse. 2010;45:700-16.

33. Rosenberg AR, Bona K, Wharton CM, Bradford M, Shaffer ML, Wolfe J, et al. Adolescent and young adult patient engagement and participation in survey-based research: a report from the "resilience in adolescents and young adults with cancer" study. Pediatr Blood Cancer. 2016;63:734-6. 
34. Lankenau SE, Sanders B, Hathazi D, Bloom JJ. Recruiting and retaining mobile young injection drug users in a longitudinal study. Subst Use Misuse. 2010;45:684-99.

35. Hanna KM, Scott LL, Schmidt KK. Retention strategies in longitudinal studies with emerging adults. Clin Nurse Spec. 2014;28:41-5.

36. Crawford JA, Hargrave TM, Hunt A, Liu CC, Anbar RD, Hall GE, et al. Issues in design and implementation in an urban birth cohort study: the Syracuse AUDIT project. J Urban Heal. 2006;83:741-59.

37. Hughes JR, Naud S, Budney AJ, Fingar JR, Callas PW. Attempts to stop or reduce daily cannabis use: an intensive natural history study. Psychol Addict Behav. 2016;30:389-97.

38. Langley JD, Lilley R, Wilson S, Derrett S, Samaranayaka A, Davie G, et al. Factors associated with non-participation in one or two follow-up phases in a cohort study of injured adults. Inj Prev. 2013;19:428-33.

39. Gabbe BJ, Cameron PA, Hannaford AP, Sutherland AM, McNeil JJ. Routine follow up of major trauma patients from trauma registries: what are the outcomes? J Trauma. 2006:61:1393-9.

40. Dorn JM, Genco RJ, Grossi SG, Falkner KL, Hovey KM, lacoviello L, et al. Periodontal disease and recurrent cardiovascular events in survivors of myocardial infarction (MI): the Western New York acute MI study. J Periodontol. 2010;81:502-11.

41. Pescud M, Pettigrew S, Wood L, Henley N. Insights and recommendations for recruitment and retention of low socio-economic parents with overweight children. Int J Soc Res Methodol. 2015;18:617-33.

42. Loue S, Sajatovic M. Research with severely mentally ill Latinas: successful recruitment and retention strategies. J Immigr Minor Health. 2008;10:145-53.

43. Wu C, Thompson ME, Fong GT, Jiang Y, Yang Y, Feng G, et al. Methods of the International Tobacco Control (ITC) China survey: waves 1, 2 and 3. Tob Control. 2015;24:iv1-5.

44. Højmark K, Støttrup C, Carreon L, Andersen MO. Patient-reported outcome measures unbiased by loss of follow-up. Single-center study based on DaneSpine, the Danish spine surgery registry. Eur Spine J. 2016;25:282-6.

45. Tansey CM, Matté AL, Needham D, Herridge MS. Review of retention strategies in longitudinal studies and application to follow-up of ICU survivors. Intensive Care Med. 2007;33:2051-7.

46. Donald M, Dower J, Ware R, Mukandi B, Parekh S, Bain C. Living with diabetes: rationale, study design and baseline characteristics for an Australian prospective cohort study. BMC Public Health. 2012;12:8.

47. McSweeney JC, Pettey CM, Fischer EP, Spellman A. Going the distance. Res Gerontol Nurs. 2009;2:256-64

48. Dubé K, Zango A, Van De Wijgert J, Meque I, Ferro JJ, Cumbe F, et al. HIV incidence in a cohort of women at higher risk in Beira, Mozambique: prospective study 2009-2012. PLoS One. 2014;9:1-6.

49. Heppner PP, Armer JM, Mallinckrodt B. Problem-solving style and adaptation in breast cancer survivors: a prospective analysis. J Cancer Surviv. 2009;3:128-36.

50. Friedman R, Bastos F, Leite I, Veloso V, Moreira R, Cardoso S, et al. Pregnancy rates and predictors in women with HIV/AIDS in Rio de Janeiro, Southeastern Brazil. Rev Saude Publica. 2011;45:373-81.

51. Albus M, Hubmann W, Mohr F, Hecht S, Hinterberger-Weber P, Seitz NN, et al. Neurocognitive functioning in patients with first-episode schizophrenia: results of a prospective 5-year follow-up study. Eur Arch Psychiatry Clin Neurosci. 2006;256:442-51.

52. Kiwanuka N, Mpendo J, Nalutaaya A, Wambuzi M, Nanvubya A, Kitandwe PK, et al. An assessment of fishing communities around Lake Victoria, Uganda, as potential populations for future HIV vaccine efficacy studies: an observational cohort study. BMC Public Health. 2014;14:986.

53. Thege BK, Woodin EM, Hodgins DC, Williams RJ. Natural course of behavioral addictions: a 5-year longitudinal study. BMC Psychiatry. 2015;15:4.

54. Sinclair JMA, Hawton K, Gray A. Six year follow-up of a clinical sample of self-harm patients. J Affect Disord. 2010;121:247-52.

55. Smith JA, Wilde MH, Brasch J. Internet recruitment and retention for a 6 months' longitudinal study. J Nurs Scholarsh. 2012:44:165-70.

56. Pendlebury ST, Chen PJ, Bull L, Silver L, Mehta Z, Rothwell PM. Methodological factors in determining rates of dementia in transient ischemic attack and stroke: (i) impact of baseline selection bias. Stroke. 2015;46:641-6.

57. Coley N, Gardette V, Toulza O, Gillette-Guyonnet S, Cantet C, Nourhashemi $F$, et al. Predictive factors of attrition in a cohort of Alzheimer disease patients: the REAL.FR study. Neuroepidemiology. 2008;31:69-79.

58. Shatenstein B, Kergoat M-J, Reid I. Issues in recruitment, retention, and data collection in a longitudinal nutrition study of community-dwelling older adults with early-stage Alzheimer's dementia. J Appl Gerontol. 2008;27:267-85.
59. Ellis KA, Szoeke C, Bush Al, Darby D, Graham PL, Lautenschlager NT, et al. Rates of diagnostic transition and cognitive change at 18-month follow-up among 1,112 participants in the Australian imaging, biomarkers and lifestyle flagship study of ageing (AIBL). Int Psychogeriatr. 2014;26:543-54.

60. Newberry A, Sherwood P, Hricik A, Bradley S, Kuo J, Crago E, et al. Understanding recruitment and retention in neurological research. J Neurosci Nurs. 2010;42:47-57.

61. Dunn KM, Campbell P, Jordan KP. Long-term trajectories of back pain: cohort study with 7-year follow-up. BMJ Open. 2013;3:e003838.

62. Novljan MP, Rotar Z, Ambrozic A, Vidmar G, Tomsic M. Comparison of the performance of the different classification criteria for primary Sjogren's syndrome: a prospective cohort study. Clin Rheumatol. 2014;33:1657-64.

63. Torgalsbøen AK, Mohn C, Czajkowski N, Rund BR. Relationship between neurocognition and functional recovery in first-episode schizophrenia: results from the second year of the Oslo multi-follow-up study. Psychiatry Res. 2015;227:185-91.

64. Jourdan C, Bayen E, Bahrami S, Ghout I, Darnoux E, Azerad S, et al. Loss to follow-up and social background in an inception cohort of patients with severe traumatic brain injury. J Head Trauma Rehabil. 2016;31:E42-8.

65. Bair E, Brownstein NC, Ohrbach R, Greenspan JD, Dubner R, Fillingim RB, et al. Study protocol, sample characteristics, and loss to follow-up: the OPPERA prospective cohort study. J Pain. 2013;14:T2-19.

66. Langley J, Johnson S, Slatyer M, Skilbeck CE, Thomas M. Issues of loss to follow-up in a population study of traumatic brain injury (TBI) followed to 3 years post-trauma. Brain Inj. 2010;24:939-47.

67. Rapoport MJ, Kiss A, Feinstein A. The impact of major depression on outcome following mild-to-moderate traumatic brain injury in older adults. J Affect Disord. 2006:92:273-6.

68. Gabbe BJ, Cleland H, Watterson DM, Schrale R, McRae S, Parker C, et al. Long term outcomes data for the burns registry of Australia and New Zealand: is it feasible? Burns. 2015;41:1732-40.

69. Fenix JB, Cherlin EJ, Prigerson HG, Johnson-Hurzeler R, Kasl SV, Bradley EH. Religiousness and major depression among bereaved family caregivers: a 13-month follow-up study. J Palliat Care. 2006;22:286-92.

70. Bennett KS, Hay DA, Piek J, Pearsall-Jones J, Levy F, Martin N. The Australian twin ADHD project: current status and future directions. Twin Res Hum Genet. 2006;9:718-26.

71. Meinck F, Cluver LD, Boyes ME. Household illness, poverty and physical and emotional child abuse victimisation: findings from South Africa's first prospective cohort study. BMC Public Health. 2015;15:444.

72. Guey LT, Bromet EJ, Gluzman SF, Zakhozha V, Paniotto V. Determinants of participation in a longitudinal two-stage study of the health consequences of the Chornobyl nuclear power plant accident. BMC Med Res Methodol. 2008;8:27.

73. Graziotti AL, Hammond J, Messinger DS, Bann CM, Miller-Loncar C, Twomey $J$, et al. Maintaining participation and momentum in longitudinal research involving high-risk families. J Nurs Scholarsh. 2012;44:120-6.

74. Williams P, Chernoff M, Angelidou K, Brouwers P, Kacanek D, Deygoo N, et al. Participation and retention of youth with perinatal HIV infection in mental health research studies. J Acquir Immune Defic Syndr. 2013:63:401-9.

75. Betancourt TS, Brennan RT, Rubin-Smith JE, Fritzmaurice GM, Gilman SE. Sierra Leone's former child soldiers: a longitudinal study of risk, protective factors, and mental health. J Am Acad Child Adolesc Psychiatry. 2011;49:606-15.

76. Hinshaw SP, Owens EB, Zalecki C, Huggins SP, Montenegro-Nevado AJ, Schrodek

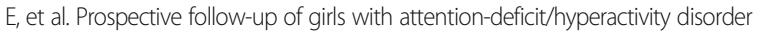
into early adulthood: continuing impairment includes elevated risk for suicide attempts and self-injury. J Consult Clin Psychol. 2012;80:1041-51.

77. Boykin DM, London MJ, Orcutt HK. Examining minority attrition among women in longitudinal trauma research. J Trauma Stress. 2016;29:26-32.

78. Johnson SB, Lynch KF, Baxter J, Lernmark B, Roth R, Simell T, et al. Predicting later study withdrawal in participants active in a longitudinal birth cohort study for 1 year: the TEDDY study. J Pediatr Psychol. 2016;41: 373-83.

79. Baral S, Kizub D, Masenior NF, Peryskina A, Stachowiak J, Stibich M, et al. Male sex workers in Moscow, Russia: a pilot study of demographics, substance use patterns, and prevalence of HIV-1 and sexually transmitted infections. AIDS Care. 2010;22:112-8.

80. Khosropour CM, Sullivan PS. Predictors of retention in an online follow-up study of men who have sex with men. J Med Internet Res. 2011:13:1-14.

81. Khosropour CM, Johnson BA, Ricca AV, Sullivan PS. Enhancing retention of an internet-based cohort study of men who have sex with men (MSM) via text messaging: randomized controlled trial. J Med Internet Res. 2013;15:1-11. 
82. Liu G, Lu H, Wang J, Xia D, Sun Y, Mi G, et al. Incidence of HIV and syphilis among men who have sex with men (MSM) in Beijing: an open cohort study. PLoS One. 2015;10:1-13.

83. Mao H, Ma W, Lu H, Wang L, Zheng H, Zhu Y, et al. High incidence of HIV and syphilis among migrant men who have sex with men in Beijing, China: a prospective cohort study. BMJ Open. 2014;4:e005351.

84. Peng Z, Yang H, Norris J, Chen X, Huan X, Yu R, et al. HIV incidence and predictors associated with retention in a cohort of men who have sex with men in Yangzhou, Jiangsu Province. China PLoS One. 2012;7:4-9.

85. Segura M, Estani SS, Marone R, Bautista CT, Pando MA, Eyzaguirre L, et al. Buenos Aires cohort of men who have sex with men: prevalence, incidence, risk factors, and molecular genotyping of HIV type 1. AIDS Res Hum Retrovir. 2007;23:1322-9.

86. Abaasa A, Asiki G, Mpendo J, Levin J, Seeley J, Nielsen L, et al. Factors associated with dropout in a long term observational cohort of fishing communities around Lake Victoria. Uganda BMC Res Notes. 2015;8:815.

87. Seed $M$, Juarez $M$, Alnatour $R$. Improving recruitment and retention rates in preventive longitudinal research with adolescent mother. J Child Adolesc Psychiatr Nurs. 2009;22:150-3.

88. Vinther-Larsen M, Riegels M, Rod MH, Schiotz M, Curtis T, Gronbaek M. The Danish youth cohort: characteristics of participants and non-participants and determinants of attrition. Scand J Public Health. 2010;38:648-56.

89. Temple JR, Choi H. Longitudinal association between teen sexting and sexual behavior. Pediatrics. 2014;134:e1287-92.

90. Orpinas $P$, Lacy B, Nahapetyan L, Dube SR, Song X. Cigarette smoking trajectories from sixth to twelfth grade: associated substance use and high school dropout. Nicotine Tob Res. 2016;18:156-62.

91. Hemphill SA, Heerde JA, Scholes-Balog KE, Herrenkohl TI, Toumbourou JW, Catalano RF. Effects of early adolescent alcohol use on mid-adolescent school performance and connection: a longitudinal study of students in Victoria, Australia and Washington state, United States. J Sch Health. 2014; 84:706-15.

92. Oldehinkel AJ, Rosmalen JGM, Buitelaar JK, Hoek HW, Ormel J, Raven D, et al. Cohort profile update: the tracking adolescents' individual lives survey (TRAILS). Int J Epidemiol. 2015;44:76.

93. Oman RF, Vesely SK, Aspy CB, Fluhr J, Marshall L, Calhoun G, et al. Methodological considerations in a community-based longitudinal study. Am J Health Behav. 2009;33:58-68.

94. Pluddemann A, Flisher AJ, McKetin R, Parry CD, Lombard CJ. A prospective study of methamphetamine use as a predictor of high school nonattendance in Cape Town, South Africa. Subst Abuse Treat Prev Policy. 2010; $5: 1-7$.

95. Van Lissa CJ, Hawk ST, Branje S, Koot HM, Meeus WHJ. Common and unique associations of adolescents' affective and cognitive empathy development with conflict behavior towards parents. J Adolesc. 2016;47: 60-70.

96. Hamdi NR, lacono WG. Lifetime prevalence and co-morbidity of externalizing disorders and depression in prospective assessment. Psychol Med. 2014:44:315-24.

97. Holt CL, Le D, Calvanelli J, Huang J, Eddie M, Roth DL, et al. Participant retention in a longitudinal national telephone survey of African American men and women. Ethn Dis. 2015;25:187-92.

98. Baird DD, Harmon QE, Upson K, Moore KR, Barker-Cummings C, Baker S, et al. A prospective, ultrasound-based study to evaluate risk factors for uterine fibroid incidence and growth: methods and results of recruitment. J Women's Heal. 2015;24:907-15.

99. Redwood D, Leston J, Asay E, Ferucci E, Etzel R, Lanier AP. Strategies for successful retention of Alaska native and American Indian study participants. J Prim Prev. 2011;32:43-52.

100. Odierna DH, Schmidt LA. The effects of failing to include hard-to-reach respondents in longitudinal surveys. Am J Public Health. 2009;99:1515-21.

101. Martin SA, Haren MT, Middleton SM, Wittert GA. The Florey Adelaide male ageing study (FAMAS): design, procedures \& participants. BMC Public Health. 2007:7:126.

102. Anders KL, Nguyen NM, Van Thuy NT, Hieu NT, Nguyen HL, Hong Tham NT, et al. A birth cohort study of viral infections in Vietnamese infants and children: study design, methods and characteristics of the cohort. BMC Public Health. 2013;13:937.

103. Panter-Brick C, Goodman A, Tol W, Eggerman M. Mental health and childhood adversities: a longitudinal study in Kabul, Afghanistan. J Am Acad Child Adolesc Psychiatry. 2011;50:349-63.
104. Hush JM, Michaleff Z, Maher CG, Refshauge K. Individual, physical and psychological risk factors for neck pain in Australian office workers: a 1-year longitudinal study. Eur Spine J. 2009;18:1532-40.

105. West R, Gilsenan A, Coste F, Zhou X, Brouard R, Nonnemaker J, et al. The ATTEMPT cohort: a multi-national longitudinal study of predictors, patterns and consequences of smoking cessation; introduction and evaluation of internet recruitment and data collection methods. Addiction. 2006;101: 1352-61.

106. Göritz AS, Wolff H-G, Goldstein DG. Individual payments as a longer-term incentive in online panels. Behav Res Methods. 2008;40:1144-9.

107. Eaton WW, Kalaydjian A, Scharfstein DO, Mezuk B, Ding Y. Prevalence and incidence of depressive disorder: the Baltimore ECA follow-up, 1981-2004 Acta Psychiatr Scand. 2007;116:182-8.

108. Ejiogu N, Norbeck JH, Mason MA, Cromwell BC, Zonderman AB, Evans MK Recruitment and retention strategies for minority or poor clinical research participants: lessons from the healthy aging in neighborhoods of diversity across the life span study. Gerontologist. 2011:51 SI:S33-45.

109. Bambs CE, Kip KE, Mulukutla SR, Aiyer AN, Johnson C, McDowell LA, et al. Sociodemographic, clinical, and psychological factors associated with attrition in a prospective study of cardiovascular prevention: the heart strategies concentrating on risk evaluation (heart SCORE) study. Ann Epidemiol. 2013;23:328-33.

110. Siukosaari P, Ajwani S, Ainamo A, Wolf J, Närhi T. Periodontal health status in the elderly with different levels of education: a 5-year follow-up study. Gerodontology. 2012;29:170-8.

111. Peat G, Thomas E, Handy J, Wood L, Dziedzic K, Myers H, et al. The knee clinical assessment study - CAS(K). a prospective study of knee pain and knee osteoarthritis in the general population: baseline recruitment and retention at 18 months. BMC Musculoskelet Disord. 2006;7:30

112. Khoo SK, O'Neill S, Travers C, Oldenburg B. Age-related changes relevant to health in women: design, recruitment, and retention strategies for the longitudinal assessment of women (LAW) study. J Womens Health (Larchmt). 2008;17:135-46.

113. Radler BT, Ryff CD. Who participates? Accounting for longitudinal retention in the MIDUS national study of health and well-being. J Aging Health. 2010; 22:307-31.

114. Stafford M, Black S, Shah I, Hardy R, Pierce M, Richards M, et al. Using a birth cohort to study ageing: representativeness and response rates in the National Survey of health and development. Eur J Ageing. 2013;10:145-57.

115. De Graaf R, Van Dorsselaer S, Tuithof M, Ten Have M. Sociodemographic and psychiatric predictors of attrition in a prospective psychiatric epidemiological study among the general population. Result of the Netherlands mental health survey and incidence Study-2. Compr Psychiatry. 2013;54:1131-9

116. Lamers F, Hoogendoorn AW, Smit JH, Van Dyck R, Zitman FG, Nolen WA, et al. Sociodemographic and psychiatric determinants of attrition in the Netherlands study of depression and anxiety (NESDA). Compr Psychiatry. 2012;53:63-70

117. Satherley N, Milojev P, Greaves LM, Huang Y, Osborne D, Bulbulia J, et al. Demographic and psychological predictors of panel attrition: evidence from the New Zealand attitudes and values study. PLoS One. 2015;10:1-22.

118. Méjean C, Szabo De Edelenyi F, Touvier M, Kesse-Guyot E, Julia C, Andreeva $V A$, et al. Motives for participating in a web-based nutrition cohort according to sociodemographic, lifestyle, and health characteristics: the nutrinet-santé cohort study. J Med Internet Res. 2014;16:e189.

119. Xie G, Laskowitz DT, Turner EL, Egger JR, Shi P, Ren F, et al. Baseline healthrelated quality of life and 10-year all-cause mortality among 1739 Chinese adults. PLoS One. 2014;9:1-9.

120. Leonard CA, Williams RJ. The relationship between gambling fallacies and problem gambling. Psychol Addict Behav. 2016;30:694-704.

121. Haring $R$, Alte $D$, Völzke $H$, Sauer $S$, Wallaschofski $H$, John $U$, et al. Extended recruitment efforts minimize attrition but not necessarily bias. J Clin Epidemiol. 2009;62:252-60.

122. Wu XM, Bennett DH, Ritz B, Tancredi DJ, Hertz-Picciotto I. Temporal variation of residential pesticide use and comparison of two survey platforms: a longitudinal study among households with young children in Northern California. Environ Health. 2013:12:65

123. Dishman RK, Vandenberg RJ, Motl RW, Nigg CR. Using constructs of the transtheoretical model to predict classes of change in regular physical activity: a multi-ethnic longitudinal cohort study. Ann Behav Med. 2010;40:150-63. 
124. Barnett W, Brittain K, Sorsdahl K, Zar HJ, Stein DJ. Maternal participant experience in a South African birth cohort study enrolling healthy pregnant women and their infants. Philos Ethics Humanit Med. 2016;11:3.

125. Loubet P, Guerrisi C, Turbelin C, Blondel B, Launay O, Bardou M, et al. First nationwide web-based surveillance system for influenza-like illness in pregnant women: participation and representativeness of the French GGrippeNet cohort. BMC Public Health. 2016;16:253

126. Martini J, Wittich J, Petzoldt J, Winkel S, Einsle F, Siegert J, et al. Maternal anxiety disorders prior to conception, psychopathology during pregnancy and early infants' development: a prospective-longitudinal study. Arch Womens Ment Health. 2013;16:549-60.

127. Saiepour N, Ware R, Najman J, Baker P, Clavarino A, Williams G. Do participants with different patterns of loss to follow-up have different characteristics? A multi-wave longitudinal study. J Epidemiol. 2016;26:45-9.

128. Daniels JL, Savitz DA, Bradley C, Dole N, Evenson KR, Eucker B, et al. Attitudes toward participation in a pregnancy and child cohort study. Paediatr Perinat Epidemiol. 2006;20:260-6.

129. Althuizen E, van Poppel MN, de Vries JH, Seidell JC, van Mechelen W. Postpartum behaviour as predictor of weight change from before pregnancy to one year postpartum. BMC Public Health. 2011;11:165.

130. Milgrom J, Gemmill AW, Bilszta JL, Hayes B, Barnett B, Brooks J, et al. Antenatal risk factors for postnatal depression: a large prospective study. J Affect Disord. 2008;108:147-57.

131. Saadeh FB, Clark MA, Rogers ML, Linkletter CD, Phipps MG, Padbury JF, et al. Pregnant and moving: understanding residential mobility during pregnancy and in the first year of life using a prospective birth cohort. Matern Child Health J. 2013:17:330-43.

132. Baird DD, Saldana TM, Shore DL, Hill MC, Schectman JM. A single baseline ultrasound assessment of fibroid presence and size is strongly predictive of future uterine procedure: 8-year follow-up of randomly sampled premenopausal women aged 35-49 years. Hum Reprod. 2015;30:2936-44.

133. Kandula NR, Puri-Taneja A, Victorson DE, Dave SS, Kanaya AM, Huffman MD. Mediators of atherosclerosis in south Asians living in America: use of webbased methods for follow-up and collection of patient-reported outcome measures. JMIR Res Protoc. 2016;5:e95.

134. Elbogen EB, Johnson SC, Newton VM, Timko C, Vasterling JJ, Van Male LM, et al. Protective mechanisms and prevention of violence and aggression in veterans. Psychol Serv. 2014;11:220-8.

135. Powers J, Tavener M, Graves A, Loxton D. Loss to follow-up was used to estimate bias in a longitudinal study: a new approach. J Clin Epidemiol. 2015;68:870-6.

136. Young AF, Powers JR, Bell SL. Attrition in longitudinal studies: who do you lose? Aust N Z J Public Health. 2006;30:353-61.

137. Decker KM. Retention of screened women in program. Can J Public Heal. 2008:99:216-20.

138. Boucher S, Gray A, Leong SL, Sharples H, Horwath C. Token monetary incentives improve mail survey response rates and participant retention: results from a large randomised prospective study of mid-age New Zealand women. N Z Med J. 2015;128:1175-8716.

139. Ekman A, Klint Å, Dickman PW, Adami HO, Litton JE. Optimizing the design of web-based questionnaires - experience from a population-based study among 50,000 women. Eur J Epidemiol. 2007;22:293-300.

140. Zielinski R, Ackerson K, Misiunas RB, Miller JM. Feasibility of a longitudinal study of women anticipating first pregnancy and assessed by multiple pelvic exams: recruitment and retention challenges. Contemp Clin Trials. 2010;31:544-8.

141. Farrall S, Hunter B, Sharpe G, Calverley A. What 'works' when retracing sample members in a qualitative longitudinal study? Int J Soc Res Methodol. 2016;19:287-300.

142. Lawrance M, Sayers SM, Singh GR. Challenges and strategies for cohort retention and data collection in an indigenous population: Australian aboriginal birth cohort. BMC Med Res Methodol. 2014;14:31.

143. Richter LM, Panday S, Norris SA. Factors influencing enrolment: a case study from birth to twenty, the 1990 birth cohort in Soweto-Johannesburg. Eval Program Plann. 2009;32:197-203.

144. Greene N, Greenland S, Olsen J, Nohr EA. Estimating bias from loss to followup in the Danish National Birth Cohort. Epidemiology. 2011;22:815-22.

145. Nunes MA, Ferri CP, Manzolli P, Soares RM, Drehmer M, Buss C, et al. Nutrition, mental health and violence: from pregnancy to postpartum cohort of women attending primary care units in southern Brazil - ECCAGE study. BMC Psychiatry. 2010;10:66.

146. Ng SK, Scott R, Scuffham PA. Contactable non-responders show different characteristics compared to lost to follow-up participants: insights from an Australian longitudinal birth cohort study. Matern Child Health J. 2016;20:1472-84.

147. Cubbin C. Survey methodology of the geographic research on wellbeing (GROW) study. BMC Res Notes. 2015;8:402.

148. Morton SMB, Grant CC, Carr PEA, Robinson EM, Kinloch JM, Fleming CJ, et al. How do you recruit and retain a prebirth cohort? Lessons learnt from growing up in New Zealand. Eval Health Prof. 2014;37:411-33.

149. Yamakawa N, Koike H, Ohtani N, Bonno M, Tanaka S, Ido M, et al. Mission in Sukusuku cohort, Mie: focusing on the feasibility and validity of methods for enrolling and retaining participants. J Epidemiol. 2010; 20(Suppl 2):S407-12.

150. Firestone R, Cheng S, Pearce N, Douwes J, Merletti F, Pizzi C, et al. Internet-based birth-cohort studies: is this the future for epidemiology? JMIR Res Protoc. 2015;4:e71.

151. Dubé E, Bettinger JA, Halperin B, Bradet R, Lavoie F, Sauvageau C, et al. Determinants of parents' decision to vaccinate their children against rotavirus: results of a longitudinal study. Health Educ Res. 2012;27:1069-80.

152. Launes J, Hokkanen L, Laasonen M, Tuulio-Henriksson A, Virta M, Lipsanen J, et al. Attrition in a 30-year follow-up of a perinatal birth risk cohort: factors change with age. PeerJ. 2014;2:e480.

153. Gartrell N, Bos H. US national longitudinal lesbian family study: psychological adjustment of 17-year-old adolescents. Pediatrics. 2010;126:28-36.

154. Brannon EE, Kuhl ES, Boles RE, Aylward BS, Benoit Ratcliff M, Valenzuela JM, et al. Strategies for recruitment and retention of families from low-income, ethnic minority backgrounds in a longitudinal study of caregiver feeding and child weight. Child Heal Care. 2013;42:198-213.

155. Baker LA, Tuvblad C, Wang P, Gomez K, Bezdjian S, Niv S, et al. The Southern California twin register at the University of Southern California: III. Twin Res Hum Genet. 2013;16:336-43.

156. Winding TN, Andersen JH, Labriola M, Nohr EA. Initial non-participation and loss to follow-up in a Danish youth cohort: implications for relative risk estimates. J Epidemiol Community Health. 2014;68:137-44.

157. Trang NHHD, Hong TK, Dibley MJ. Cohort profile: Ho Chi Minh City youth cohort--changes in diet, physical activity, sedentary behaviour and relationship with overweight/obesity in adolescents. BMJ Open. 2012;2:e000362.

158. Strycker LA, Duncan SC, Duncan TE, He H, Desai N. Retention of AfricanAmerican and white youth in a longitudinal substance use study. J Ethn Subst Abus. 2006:5:119-31.

159. Whitbeck LB, Sittner Hartshorn KJ, Crawford DM, Walls ML, Gentzler KC, Hoyt DR. Mental and substance use disorders from early adolescence to young adulthood among indigenous young people: final diagnostic results from an 8-year panel study. Soc Psychiatry Psychiatr Epidemiol. 2014:49:961-73.

160. Neiswanger K, McNeil DW, Foxman B, Govil M, Cooper ME, Weyant RJ, et al. Oral health in a sample of pregnant women from northern Appalachia (2011-2015). Int J Dent. 2015;2015:1-12.

161. Strotmeyer ES, Arnold AM, Boudreau RM, Ives DG, Cushman M, Robbins $J A$, et al. Long-term retention of older adults in the cardiovascular health study: implications for studies of the oldest old. J Am Geriatr Soc. 2010;58:696-701

162. Gu D. General Data Quality Assessment of the CLHLS. In: Zeng Y, Poston Jr DL, Ashbaugh Vlosky D, Gu D, editors. Healthy Longevity in China: Demographic, Socioeconomic, and Psychological Dimensions. Dordrecht: Springer Science+Business Media B.V.; 2008. p. 39-60.

163. Stevens NL, Van Tilburg TG. Cohort differences in having and retaining friends in personal networks in later life. J Soc Pers Relat. 2011;28:24-43.

164. Adams ER, Nolan VG, Andersen SL, Perls TT, Terry DF. Centenarian offspring: start healthier and stay healthier. J Am Geriatr Soc. 2008;56:2089-92.

165. Davies K, Kingston A, Robinson L, Hughes J, Hunt JM, Barker SAH, et al. Improving retention of very old participants in longitudinal research: experiences from the Newcastle 85+ study. PLoS One. 2014;9:1-10.

166. Carlson BW, Carlson JR, Neelon VJ, Hartman M. Tailoring protocols to successfully recruit and retain older adults in a longitudinal study of sleep and cognition. Res Gerontol Nurs. 2008;1:232-7.

167. Allman RM, Sawyer P, Crowther M, Strothers HS, Turner T, Fouad MN. Predictors of 4-year retention among African American and white community-dwelling participants in the UAB study of aging. Gerontologist. 2011:51:S46-58.

168. Gerhart JI, Hall BJ, Russ EU, Canetti D, Hobfoll SE. Sleep disturbances predict later trauma-related distress: cross-panel investigation amidst violent turmoil. Health Psychol. 2014;33:365-72. 
169. Tay A, Rees S, Steel Z, Tam N, Soares Z, Soares C, et al. Six-year trajectories of post-traumatic stress and severe psychological distress symptoms and associations with timing of trauma exposure, ongoing adversity and sense of injustice: a latent transition analysis of a community cohort in conflictaffected Ti. BMJ Open. 2016;6:e010205.

170. Walker J, Fairley CK, Urban E, Chen MY, Bradshaw C, Walker SM, et al.

Maximising retention in a longitudinal study of genital chlamydia trachomatis among young women in Australia. BMC Public Health. 2011;11:156.

Ready to submit your research? Choose BMC and benefit from:

- fast, convenient online submission

- thorough peer review by experienced researchers in your field

- rapid publication on acceptance

- support for research data, including large and complex data types

- gold Open Access which fosters wider collaboration and increased citations

- maximum visibility for your research: over $100 \mathrm{M}$ website views per year

At $\mathrm{BMC}$, research is always in progress.

Learn more biomedcentral.com/submissions 